\title{
Robust Automatic Identification of Microplastics in Environmental Samples using FTIR Microscopy - Supporting Information
}

\author{
Gerrit Renner, ${ }^{\dagger, \ddagger}$ Philipp Sauerbier, $₫$ Torsten C. Schmidt, ${ }^{\ddagger}$ Jürgen Schram ${ }^{*} \dagger$
}

$\dagger$ Instrumental Analytical and Environmental Chemistry, Faculty of Chemistry, Niederrhein University of Applied Sciences, Frankenring 20, D-47798 Krefeld, Germany

$\ddagger$ Instrumental Analytical Chemistry and Centre for Water and Environmental Research (ZWU), University of DuisburgEssen, Universitätsstr. 5, D-45141 Essen, Germany

I Wood Biology and Wood Products, Faculty of Forest Sciences, University of Goettingen, Büsgenweg 4, D37077 Göttingen, Germany

E-mail: juergen.schram@hs-niederrhein.de

Phone: +49 (0)179 1043284

\section{Contents}

1 Definitions, Figures and Tables $\quad 2$

1.1 In-House Reference Spectra Library . . . . . . . . . . . . . . . . . . 2

1.2 Examined Library Searching Algorithms . . . . . . . . . . . . . . . . . 3

1.3 Spectra of Case Study $1 \ldots \ldots \ldots \ldots \ldots \ldots$

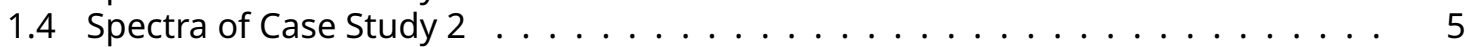

1.5 Wood-Polymer-Composite Manufacturing . . . . . . . . . . . . . . . . 6

1.6 Optimization of Data Pre-Processing Steps . . . . . . . . . . . . . . . . . 7

1.7 Smoothing with Variable Span . . . . . . . . . . . . . . . . . . . . . 8

1.8 An example of how the comparison algorithm works? . . . . . . . . . . . . . . . 99

1.9 HQI-Microplastics Amount Relation of Case Study $2 \ldots \ldots$. . . . . . . . . . . . 10

1.10 Identification Results of Case Study $3 \ldots \ldots \ldots \ldots \ldots$

2 Pyhton Source Code $\quad 12$

2.1 Create Main Window . . . . . . . . . . . . . . . . . . . . . . . . . . 12

2.2 Import Module . . . . . . . . . . . . . . . . . . . . . . . 13

2.3 Job Module . . . . . . . . . . . . . . . . . . . . . . . . . . . . . 14

2.4 Curve Fit Module . . . . . . . . . . . . . . . . . . . . . . . . 15

2.5 Database Module . . . . . . . . . . . . . . . . . . . . . . . . . 16

2.6 Library Search Module . . . . . . . . . . . . . . . . . . . . . . . . . . . . . 17

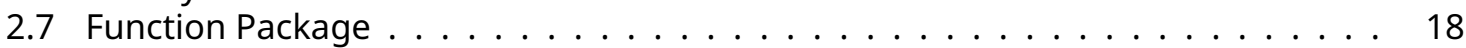

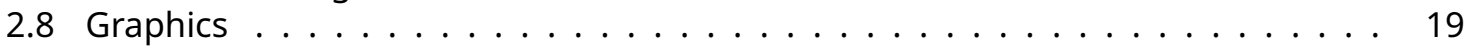




\section{In-House Reference Spectra Library}

Table S 1: Overview of the In-House Reference Database. All Materials were measured with FTIR ATR and FTIR microscopy. For identification, ATR data were compared to the ATR spectra library and $\mu$ FTIR data were compared to the $\mu$ FTIR spectra library.

\begin{tabular}{|c|c|c|c|c|}
\hline Polymer & Label & Shape & Diameter & Source \\
\hline Polyethylene & LDPE & granules & $5 \mathrm{~mm}$ & Carat $\mathrm{GmbH}$ \\
\hline Polypropylene & PP & granules & $5 \mathrm{~mm}$ & Carat $\mathrm{GmbH}$ \\
\hline Polyamide & PA-6 & granules & $5 \mathrm{~mm}$ & Carat $\mathrm{GmbH}$ \\
\hline Polyvinyl chloride & PVC & powder & $0.1 \mathrm{~mm}$ & Solvay \\
\hline Polycarbonate & PC & granules & $5 \mathrm{~mm}$ & Covestro AG \\
\hline Polyurethane & PU & flakes & $20 \mathrm{~mm}$ & $\begin{array}{l}\text { Carl Bernh. Hoffmann } \\
\text { GmbH \& Co. KG }\end{array}$ \\
\hline Polystyrene & PS & film & $0.1 \mathrm{~mm}$ & Thermo Fisher Scientific Inc \\
\hline Polyethylene terephthalate & PET & granules & $5 \mathrm{~mm}$ & Carat $\mathrm{GmbH}$ \\
\hline Polymethyl methacrylate & PMMA & powder & $0.5 \mathrm{~mm}$ & acrylic glass sheet (milled) \\
\hline Chitin & Chitin & powder & $0.5 \mathrm{~mm}$ & shrimps (shells milled) \\
\hline Polybutadiene & $\mathrm{BR}$ & fragments & $0.5 \mathrm{~mm}$ & car tires (milled) \\
\hline Polylactide & PLA & powder & $0.5 \mathrm{~mm}$ & 3D printer filament (milled) \\
\hline Polyoxymethylene & POM & powder & $0.5 \mathrm{~mm}$ & $\begin{array}{l}\text { conical joint clips (milled), } \\
\text { Carl Roth } \mathrm{GmbH}+\text { Co. KG }\end{array}$ \\
\hline Polytetrafluoroethylene & PTFE & flakes & $1 \mathrm{~mm}$ & thread sealing tape \\
\hline Celluloid & Celluloid & flakes & $1 \mathrm{~mm}$ & photography film \\
\hline Cellulose Acetate & CA & fibers & $1 \mathrm{~mm}$ & cigarette filters \\
\hline Wood-plastic composite $\left(\mathrm{PP}_{30 \% \mathrm{wt} .}\right)$ & WPC & granules & $5 \mathrm{~mm}$ & $\begin{array}{l}\text { Wood Biology \& Products, } \\
\text { University of Goettingen }\end{array}$ \\
\hline Parafilm & Paraffin wax / PE & flakes & $1 \mathrm{~mm}$ & Carl Roth GmbH + Co. KG \\
\hline Natural Rubber & Rubber & fragments & $0.5 \mathrm{~mm}$ & natural rubber (milled) \\
\hline Neoprene & Neoprene & flakes & $1 \mathrm{~mm}$ & $\begin{array}{l}\text { laboratory gloves, } \\
\text { Carl Roth } \mathrm{GmbH}+\mathrm{Co} . \mathrm{KG}\end{array}$ \\
\hline
\end{tabular}




\section{Examined Library Searching Algorithms}

Table S 2: Overview of the examined algorithms for hit quality index calculation. ${ }^{*}$

\begin{tabular}{|c|c|}
\hline Algorithm & Formula \\
\hline Euclidean Distance & {$\left[1+\frac{\|\mathbf{s}-\mathbf{r}\|^{2}}{\|\mathbf{r}\|^{2}}\right]^{-1}$} \\
\hline Pearson $r^{2}$ & $\frac{\mathbf{s} \cdot \mathbf{r}}{\|\mathbf{s}\|\|\mathbf{r}\|}$ \\
\hline $\begin{array}{l}\text { Pearson } r^{2} \\
1^{\text {st }} \text { Derivative }\end{array}$ & $\frac{\partial \mathbf{s} \cdot \partial \mathbf{r}}{\|\partial \mathbf{s}\|\|\partial \mathbf{r}\|}$ \\
\hline $\begin{array}{l}\text { Pearson } \mathrm{r}^{2} \\
2^{\text {nd }} \text { Derivative }\end{array}$ & $\frac{\partial^{2} \mathbf{s} \cdot \partial^{2} \mathbf{r}}{\left\|\partial^{2} \mathbf{s}\right\|\left\|\partial^{2} \mathbf{r}\right\|}$ \\
\hline$\mu I D E N T$ & $1-\left(\frac{\sum d_{i} \cdot w_{i}}{\sum w_{i}}\right)^{W}$ \\
\hline
\end{tabular}




\section{Spectra of Case Study 1}
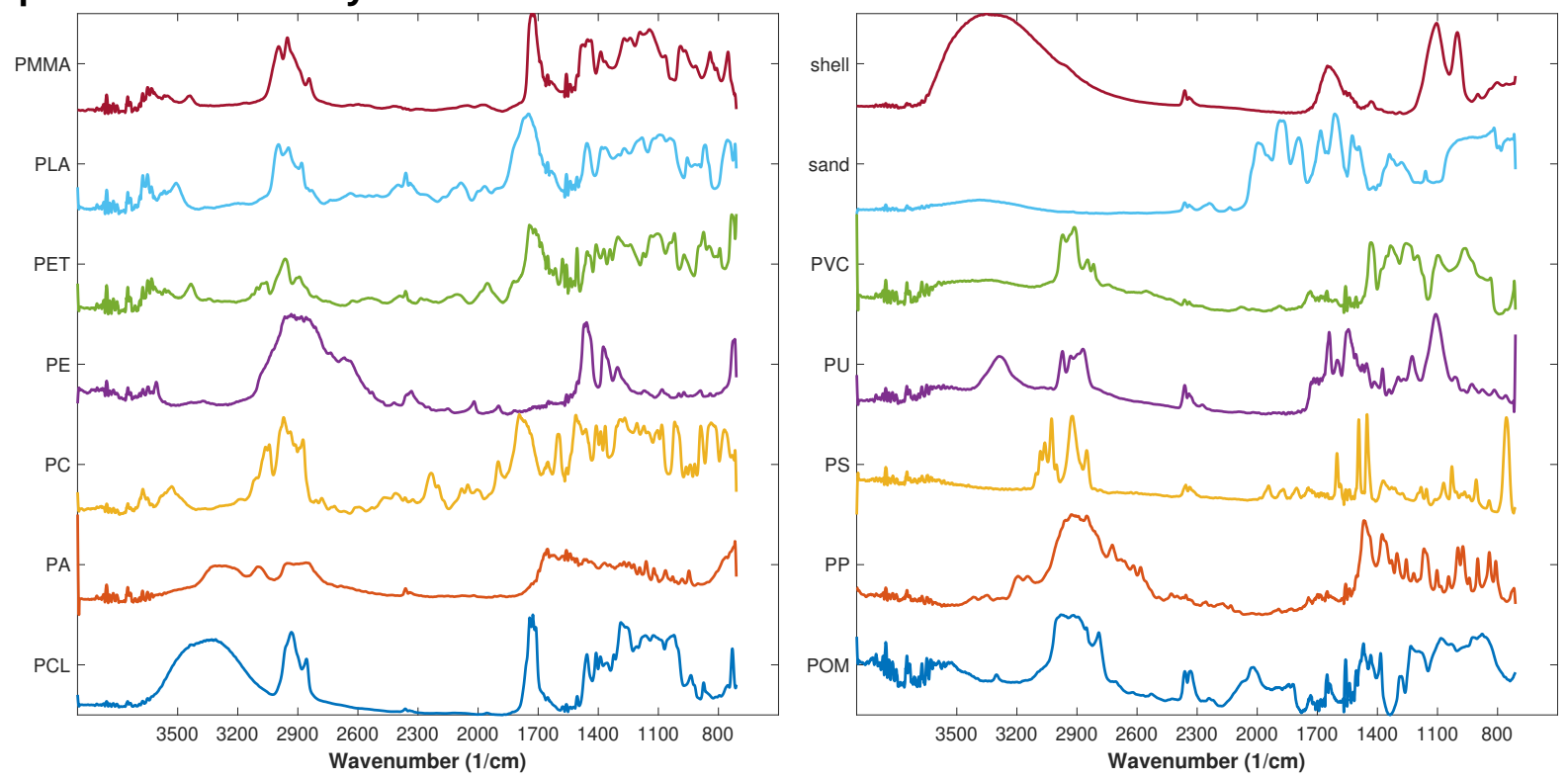

Figure S 1: Infrared microscope absorbance spectra of selected reference materials. PCL: polycaprolactone, PA: polyamide, PC: polycarbonate, PE: polyethylene, PET: polyethylene terephthalate, PLA: polylactide, PMMA: poly(methyl methacrylate), POM: polyoxymethylene, PP: polypropylene, PS: polystyrene, PU: polyurethane, PVC: polyvinyl chloride, shell limestone and sea sand particles 
Spectra of Case Study 2

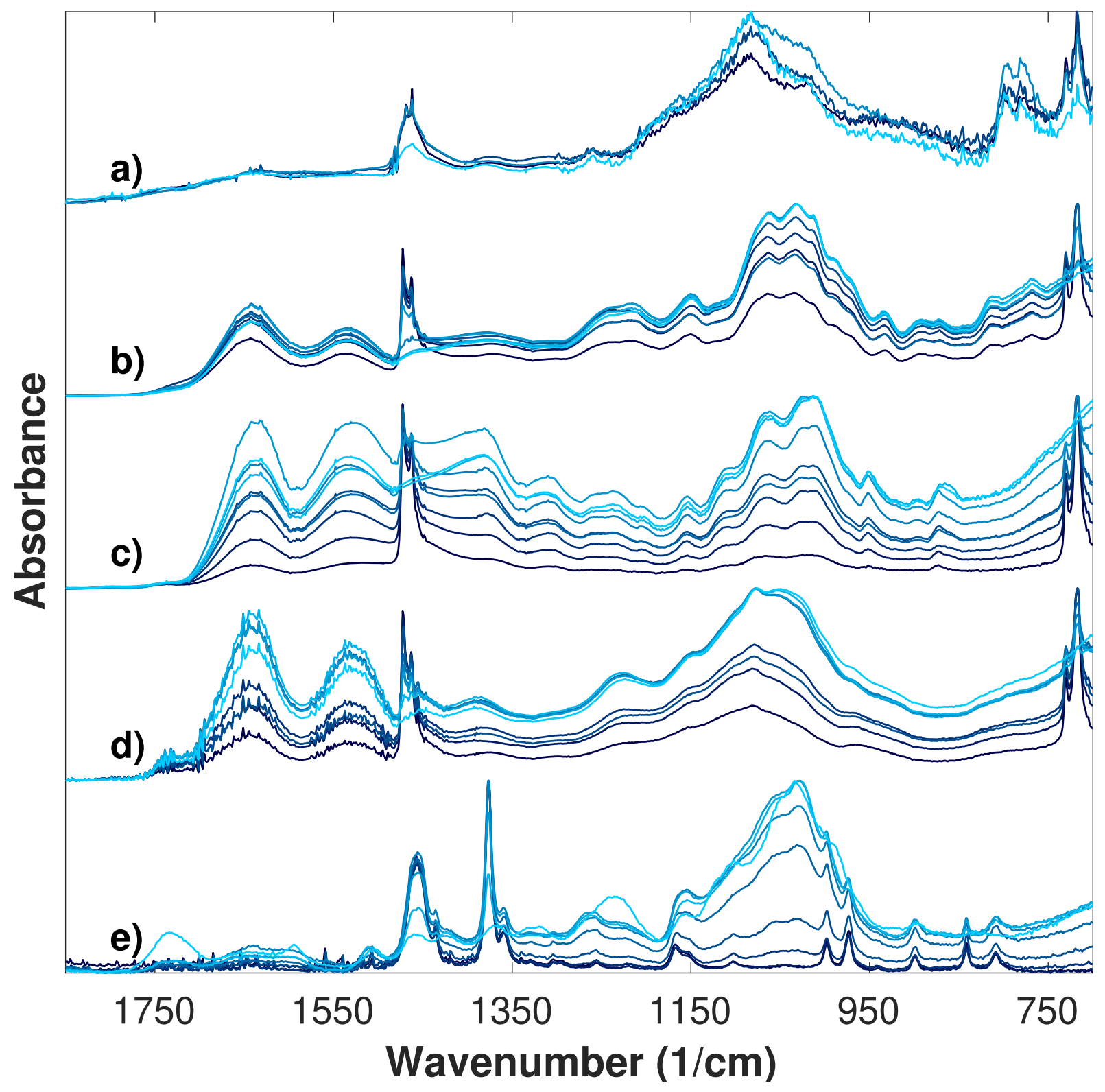

Figure S 2: FTIR ATR spectra of microplastics in presence of interfering matrices. Every mixture ranges between low (dark blue) and high (light blue) matrix content. a) PE + sand (Matrix: 20, 40, 60, $80 \%$ wt.), b) PE + algae (Porphyra) (Matrix: 17, 20, 25, 33, 50, 67, 75, 80, $83 \%$ wt.), c) PE + chitosan (Matrix: 10, 20, 30, 40, 50, 60, 70, $80,90 \%$ wt.), d) PE + algae (Chlorophyta) (Matrix: 10, 20, 30, 40, 50, 60, 70, 80, $90 \%$ wt.), e) PP + wood (spruce) (Matrix: 0, 10, 20, 30, 40, 50, 60, 70, $100 \%$ wt.). 


\section{Wood-Polymer-Composite Manufacturing}

Particles with a grain size of 70-150 $\mu$ mwere used for wood powder softwood (Arbocel C 100; J. Rettenmaier \& Söhne GmbH und Co.KG, Rosenberg, Germany). Polypropylene (PP) was used as a polymer matrix (SABIC 575P, Riyadh, Saudi Arabia). Maleic anhydride-grafted polypropylene (MAPP) as coupling agent was also added to the composites for an application-oriented and realistic investigation. The amount of coupling agent was fixed to $3 \%_{w t}$ of the PP.

The WPC was compounded in a Leistritz MICRO27GL/GG40D co-rotating twin-screw extruder (Leistritz Extrusionstechnik GmbH, Germany) with gravimetric feeders (Brabender GmbH \& Co. KG, Germany) and a hot-cut pelletizer. The granulates were produced with a mould temperature of about $140^{\circ} \mathrm{C}$. A total of eight WPC formulations were produced, with the wood content ranging from $0-70 \%$ wt in $10 \%_{w t}$ increments. 


\section{Optimization of Data Pre-Processing Steps}

The presented new modified microplastics identification algorithm is based on diverse data pre-processing steps. Thereby, the optimizations of these steps were performed using 200 FTIR microscopy spectra of microplastics reference particles made of polypropylene, polyamide and polylactide. The primary objective is to describe every FTIR spectrum by an individual cumulative pseudo Voigt function as precise as possible. To that end, a suitable data pre-processing leads to highest similarity values between pre-processed measurement data and fitted data. In other words, the error (normalized root-mean-square error, nRMSE) should become as small as possible. The optimization process is illustrated in Eq. S1

\section{Eq. S1: Optimize Data Pre-Processing}

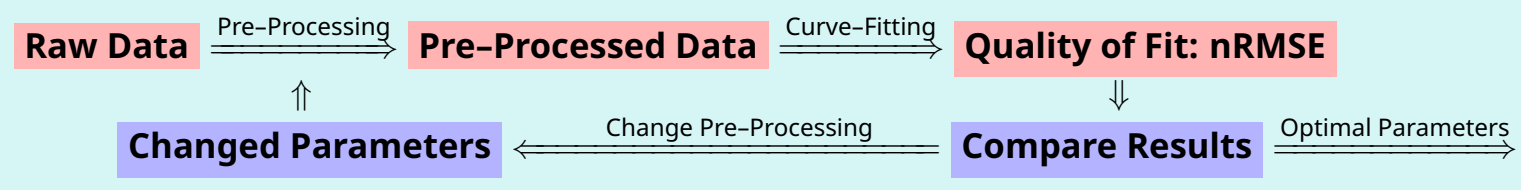

The data pre-processing optimizations were performed for two independent steps: baseline correction and data smoothing. Within both steps there were different algorithms tested and their resulting $\mathrm{nRMSE}$ values were compared with each other. However, to find optimal parameters for every individual pre-processing algorithm, Downhill-Simplex-Optimizations according to Nelder and Mead were performed focusing on the minimization of nRMSE. As input, reference spectra of PP, PA and PLA were treated by the individual algorithms with discrete parameter settings, and as output, the means of nRMSE were recorded, while the latter was used as the optimization target. Baseline correction was optimized initially and the best method was used for further optimizations of data smoothing. All examined algorithms with their best target values and optimized parameters can be seen in Table $\mathrm{S} 3$.

Table S 3: Experimental Design to Optimize Baseline Correction and Data Smoothing of Infrared Spectra of Microplastics.

\begin{tabular}{|c|c|c|c|c|}
\hline Pre-Processing Algorithm & $\begin{array}{l}\text { Polypropylene } \\
\text { nRMSE (\%) }\end{array}$ & $\begin{array}{l}\text { Polyamide } \\
\text { nRMSE (\%) }\end{array}$ & $\begin{array}{l}\text { Polylactide } \\
\text { nRMSE (\%) }\end{array}$ & $\begin{array}{l}\text { Average } \\
\text { nRMSE (\%) }\end{array}$ \\
\hline \multicolumn{5}{|l|}{ Baseline Correction } \\
\hline Rolling circle filter & 12.34 & 11.85 & 13.52 & 12.57 \\
\hline $\begin{array}{l}\text { Adaptive iteratively reweighted } \\
\text { penalized least squares smoothing (airPLS) }\end{array}$ & 9.05 & 9.44 & 9.49 & 9.32 \\
\hline Wavelet transformation & 10.22 & 11.55 & 12.03 & 11.27 \\
\hline \multicolumn{5}{|l|}{ Baseline Correction (airPLS) + Data Smoothing } \\
\hline Rolling circle filter & 5.93 & 6.00 & 6.01 & 5.98 \\
\hline $\begin{array}{l}\text { Adaptive iteratively reweighted } \\
\text { penalized least squares smoothing }\end{array}$ & 8.88 & 8.58 & 9.13 & 8.86 \\
\hline Wavelet transformation & 4.78 & 5.12 & 4.98 & 4.96 \\
\hline Moving Average & 6.84 & 7.08 & 6.65 & 6.86 \\
\hline Locally weighted scatterplot smoothing & 5.21 & 5.55 & 4.23 & 5.00 \\
\hline Savitzky-Golay filter (static smoothing span) & 3.14 & 6.12 & 3.84 & 4.37 \\
\hline Savitzky-Golay filter (variable smoothing span) & 2.67 & 5.20 & 3.16 & 3.68 \\
\hline
\end{tabular}


moothing with Variable Span

Noise levels of infrared spectra are rarely constant but often variable. One origin of this pheare no noise but the bevave very similar and disturb the measured data significantly ed signats performing water and atmosphere compensation, wh

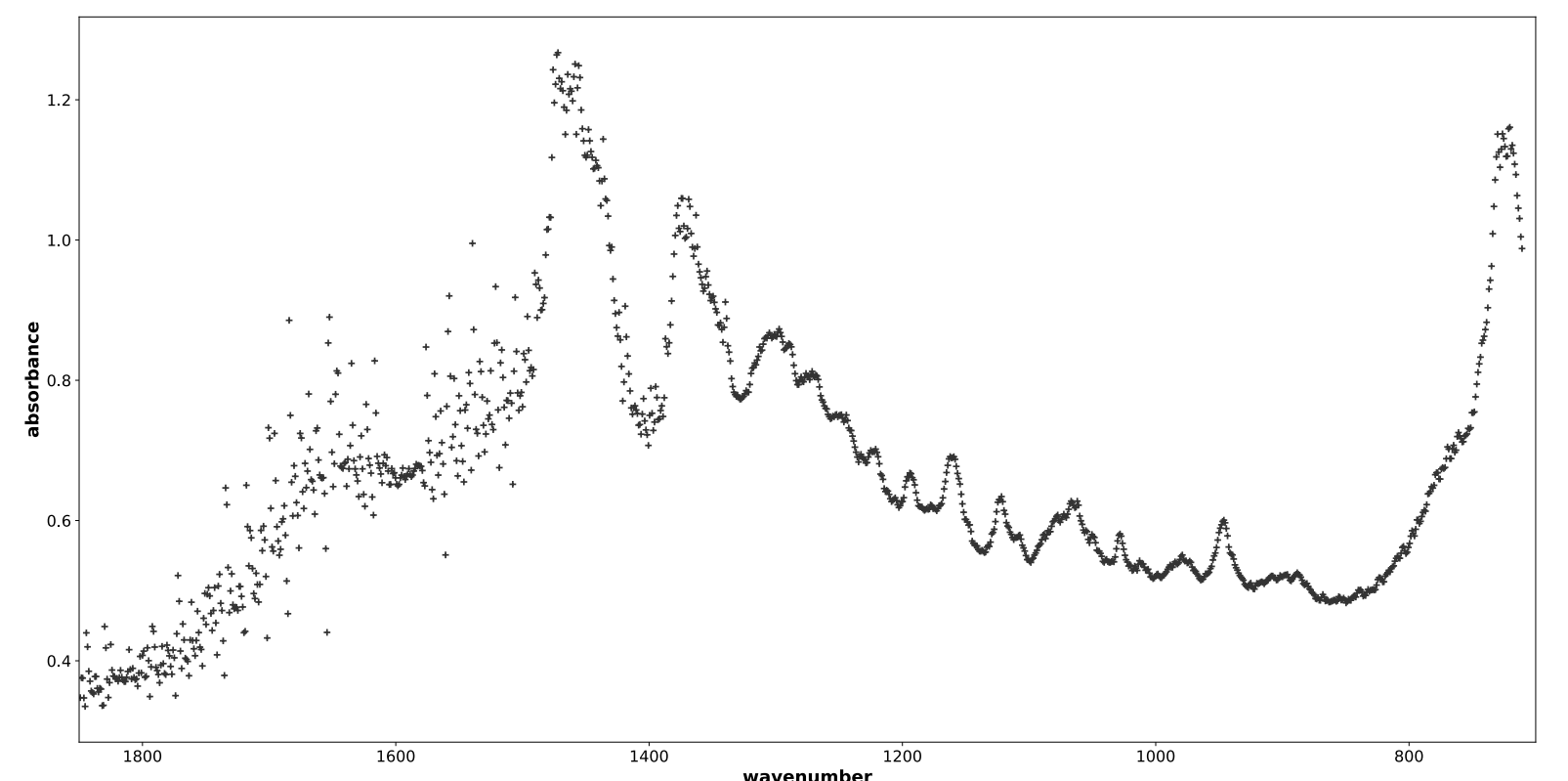

Figure 53 : urTTRs spectrum of polyethylene microplastics sfter water and atmosphere compensation. The given spectrum
still contains significant noisy disturbances, and thereffore, it has to be smoothed in further spectrum treatment.

In the shown scenario, a constant smoothing span is not suitable, as the smoothed spectrum will To that end, a smoothing method was slightly modified to work with a variable smoothing span,
which can be seen in Figure 4 .

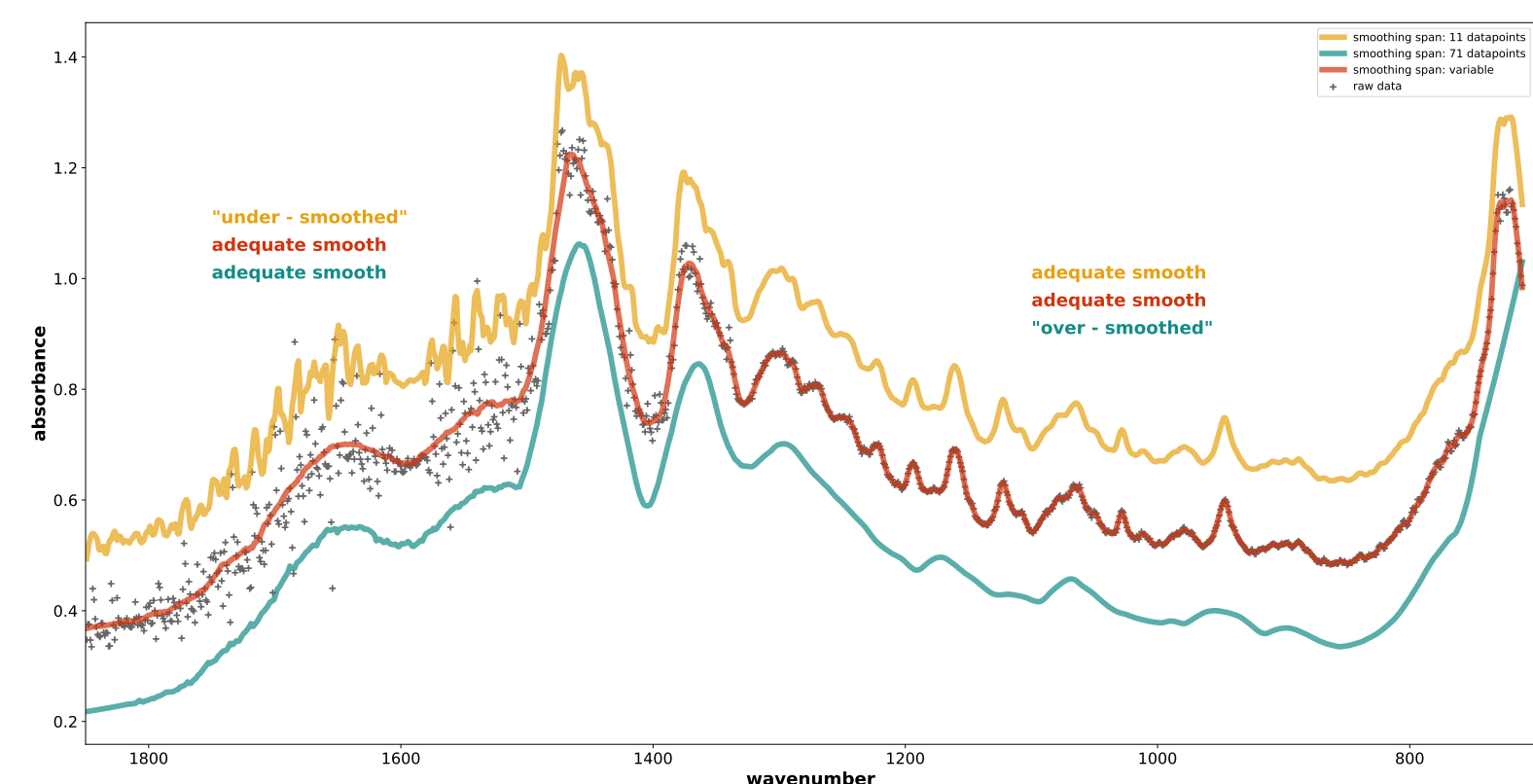

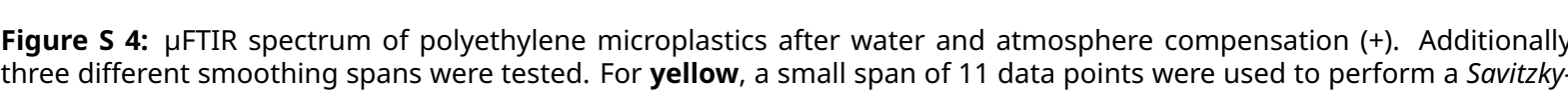

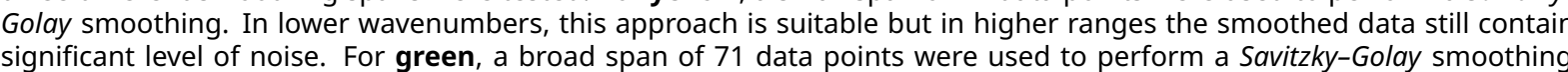

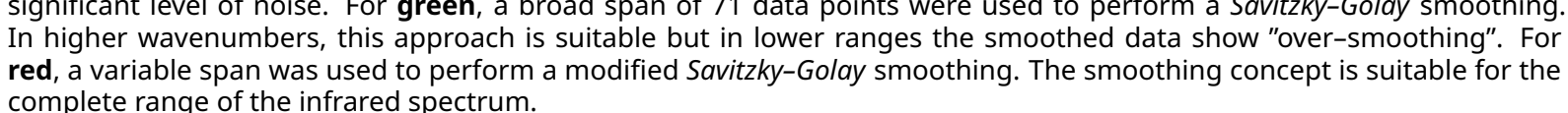
To modify the used Savitzky-Golay smoothing algorithm, the spectrum is separated into multiple
overlapping sections of 360 data points. The overlapping range is set to 60 data points, which means that every data point is smoothed 5 times with individually optimised smoothing spans,

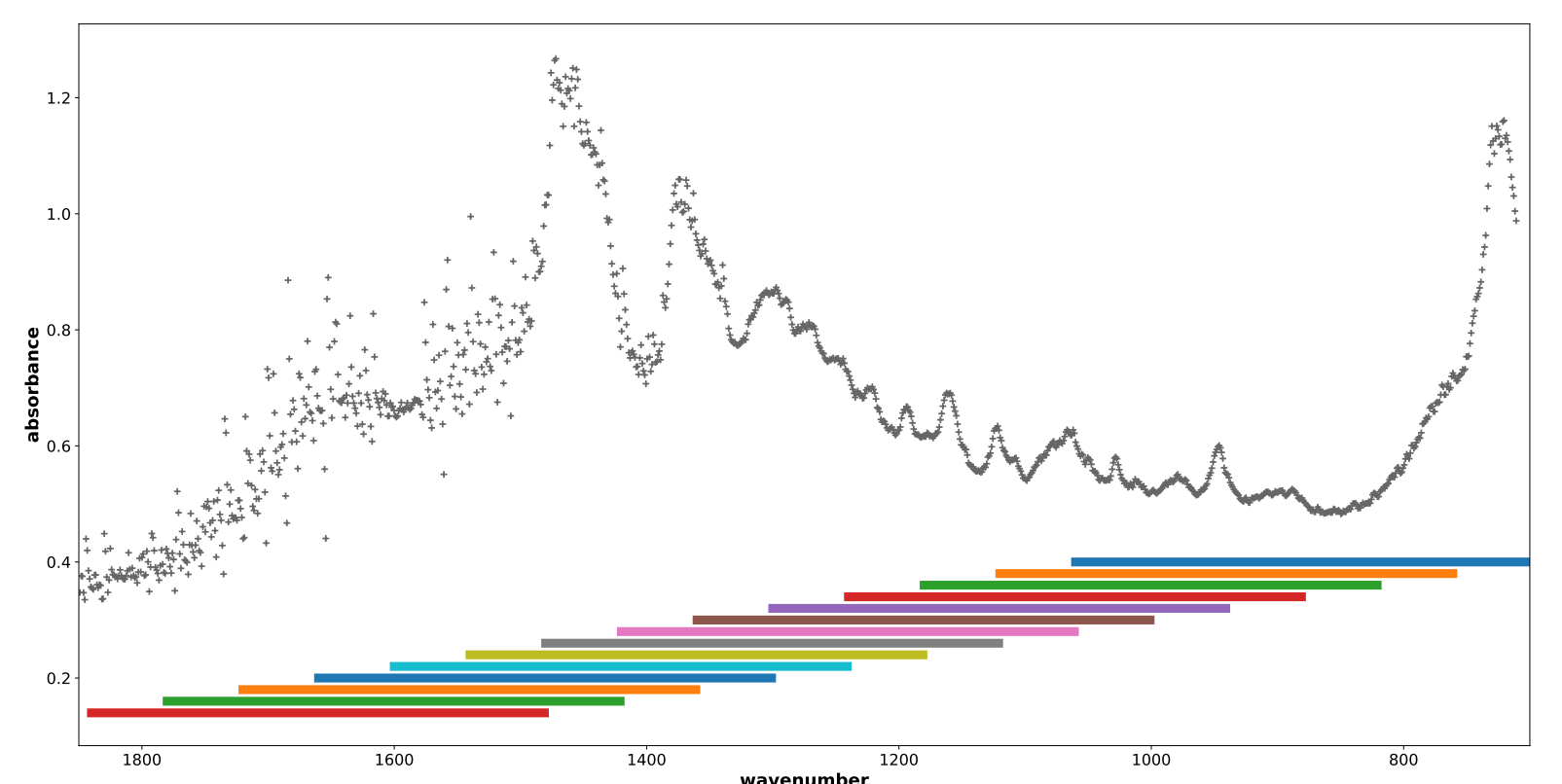

Figure 5 5: fFrTR spectrum of polyethylene $m$ m.
indicate the overlapping subdivided sections.

In a second step, each sep arated ditarange is smoothed individually by Savitzky- Goloy smo thing. However, to create a suitable and practical algorithm, finding the optimal smoothing span has to be automated, which requires a target value that describes the quality of the iteratively performed Eq. 52

Step 1: Define a function that connects smoothing span and smoothing quality. : smoothing span $\rightarrow \underbrace{\text { smoothing quality }}$

Step 2: Optimise smoothing quality $(z)$ by changing smoothing span $(w)$.

Describing the quality of a performed smoothing process is a complex task. One sulation be obtained - individual frequencies that are part of the transformed data and their amplitude. If data is noisy, many different frequencies with highly variable amplitudes will appear in the trans-
formed data set. If the data is smoothed well significantly less frequencies with smaller homogeneous amplitudes can be observed in the transformed data set This phenomenon can be used to describe a suitable target value by looking at the distribution (histogram) of the amplitudes

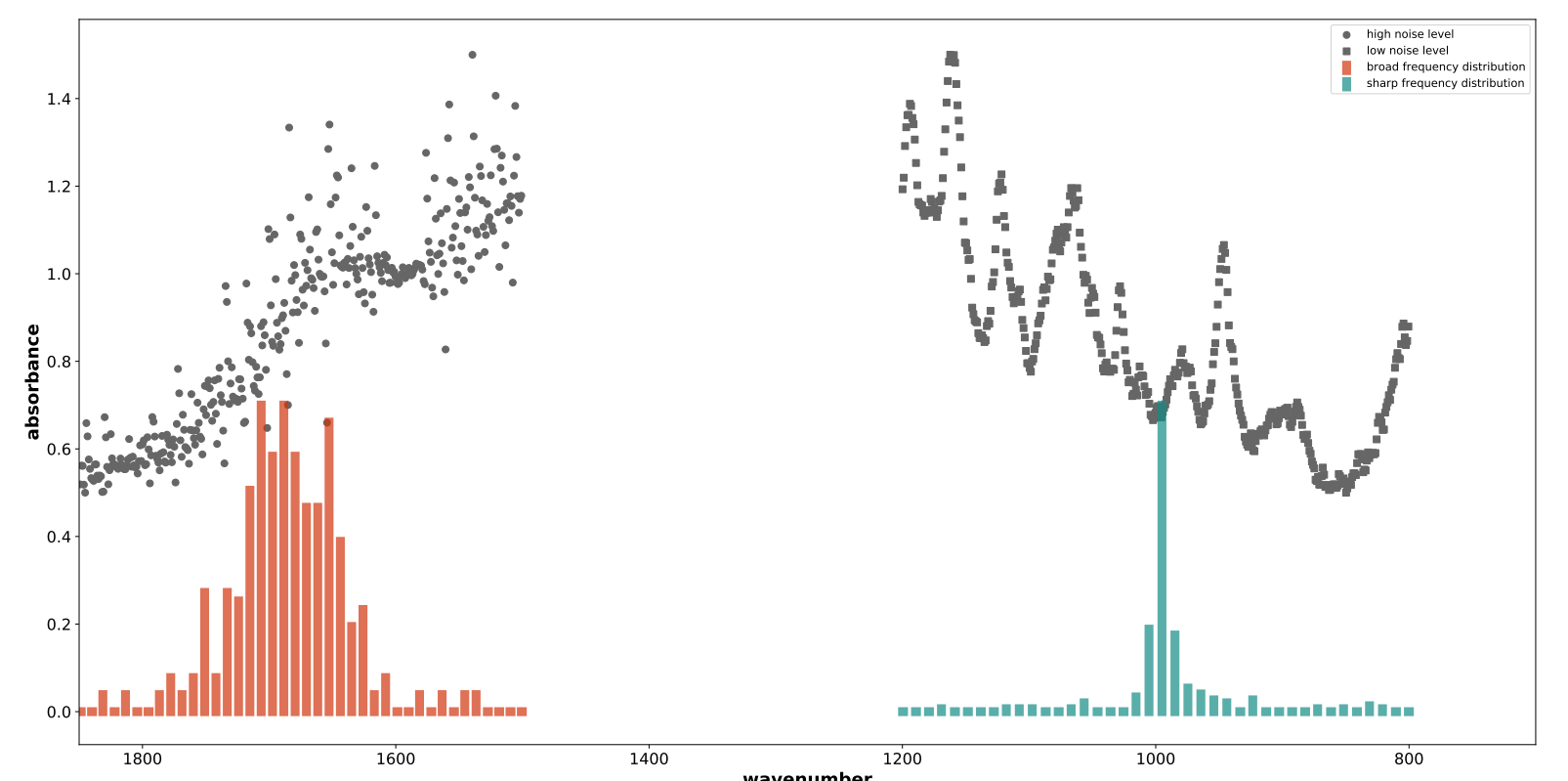

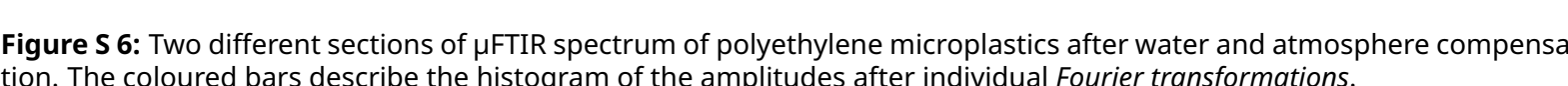
Changing iteratively the smoothing span, will also change the width of the amplitude distribution peak. If a defined threshold criterion is reached, a suitable smoothing span for the analysed section
can be extracted which can be seen in Figure $\mathbf{~}$.

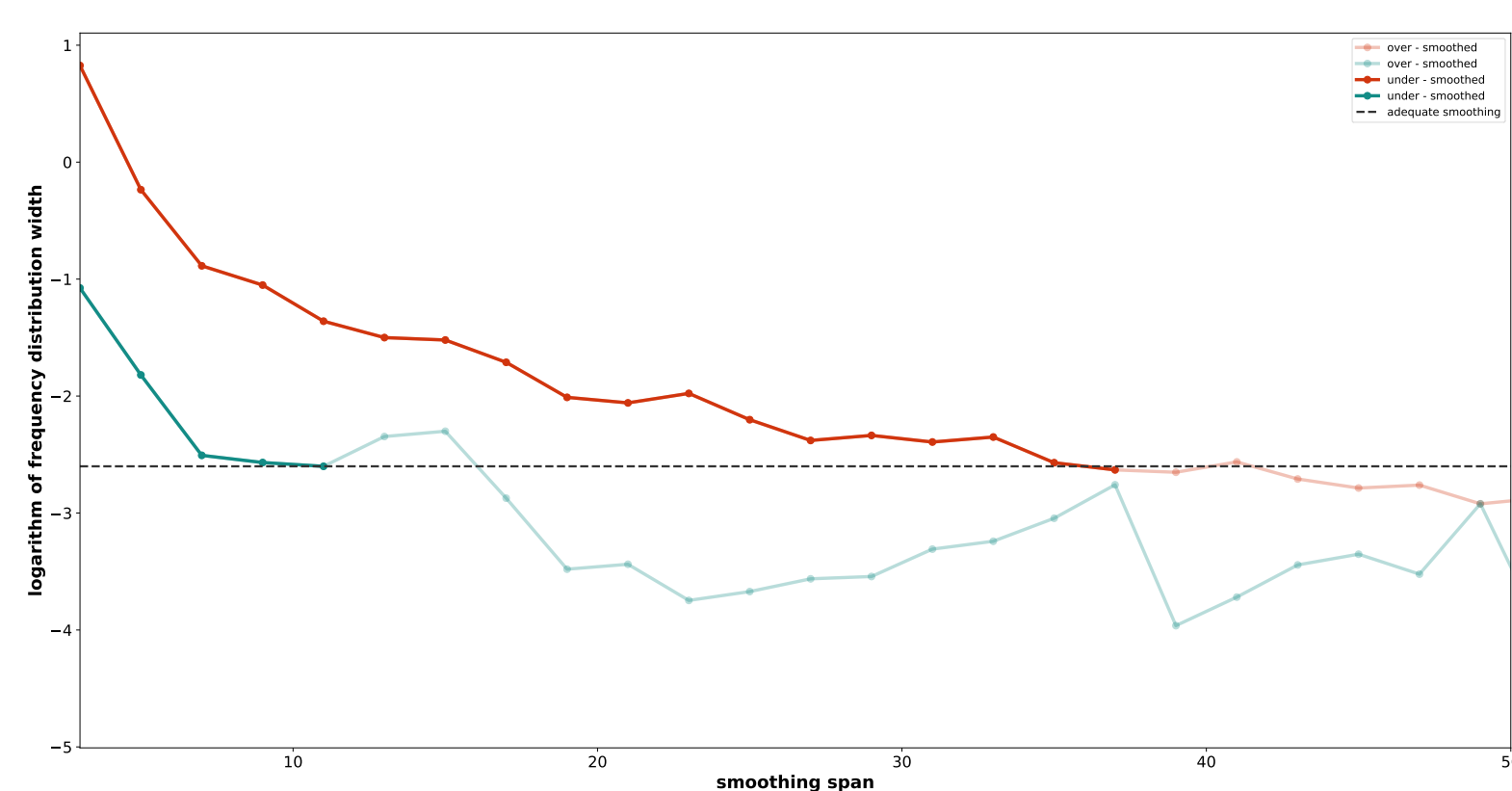

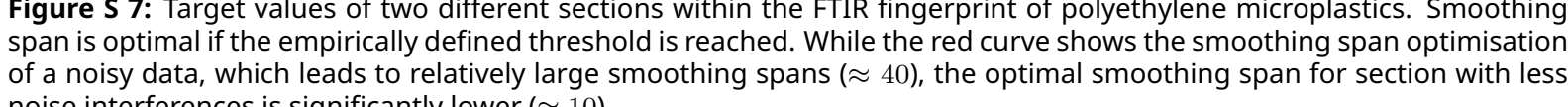

noise interferences is significanty lower $\approx$ 10). After individual smoothing span optimisation, all smoothed sections were re-concatenated. As
every data point is described by multiple smoothings caused by section overlapping, median for each wavenumber is extracted. As a result, the optimised smoothed infrared spectrum with a
variable smoothing span can be obtained. 


\section{An example of how the comparison algorithm works?}

At the end of the presented data pre-processing method the incoming raw FTIR spectra were transformed into compact lists that contain vibrational band positions, areas and weighting factors. Every sample or reference is connected to an individual list. For Hit Quality Index calculation the similarity of those lists has to be estimated, and therefore, a special algorithm was developed ${ }^{1}$, which will be explained based on a graphical example in the following. However, a more detailed description can be found in the former $\mu$ IDENT study ${ }^{1}$.

The main idea of the comparison algorithm is not to compare the vibrational band positions and areas directly but first to create a highly characteristic pattern of all unique vibrational band pairs and calculate their area ratios, which can be seen in Fig. 8 .

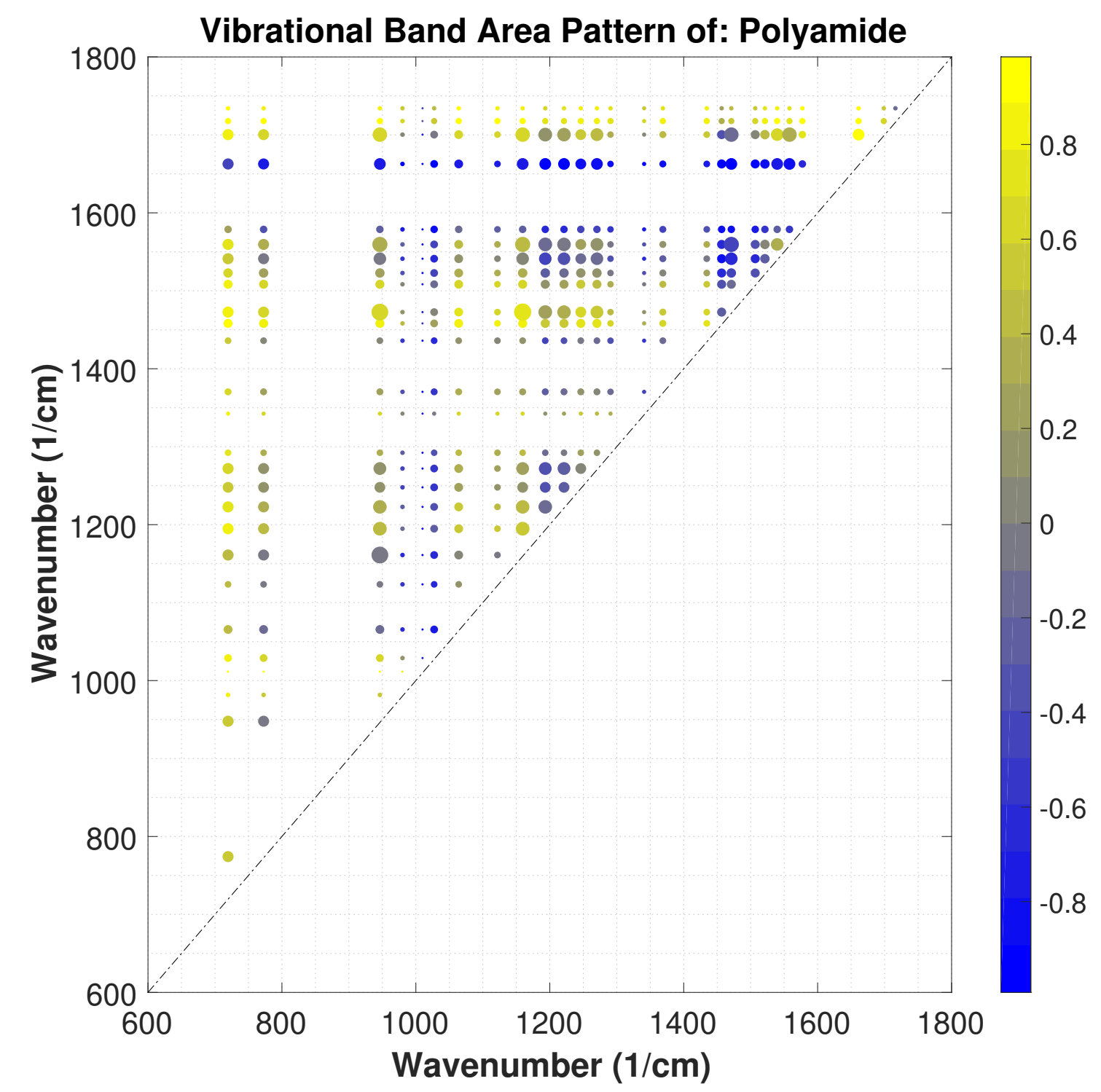

Figure S 8: Vibrational Band Area Pattern of Polyamide. The Scatter plot shows multiple colored different sized circles. Every circle represents an individual vibrational band pair within the FTIR spectrum of polyamide. The x/y coordinates are connected to the band pair positions. The circle color is the virtual third dimension of this diagram and represents the normalized area ratio of the band pair, which was calculated as the area difference divided by the area sum. The circle size represents the weighting factor of the individual band pair, which is mainly influenced by the vibrational band heights and the goodnesses of fit. Typically, the outer form of the pattern can be reduced to a triangle, due to symmetric duplicates.

For library search, the vibrational band area patterns of sample and reference were compared. To that end, all matching band pairs of reference and sample were extracted, and the differences of their positions and area ratios were averaged under the consideration of the weighting factors. For a high Hit Quality Index, the major part of all reference signals could be found within the sample pattern as well, and the differences between the area ratios were very small, which can be obtained from Fig. 9.

a)

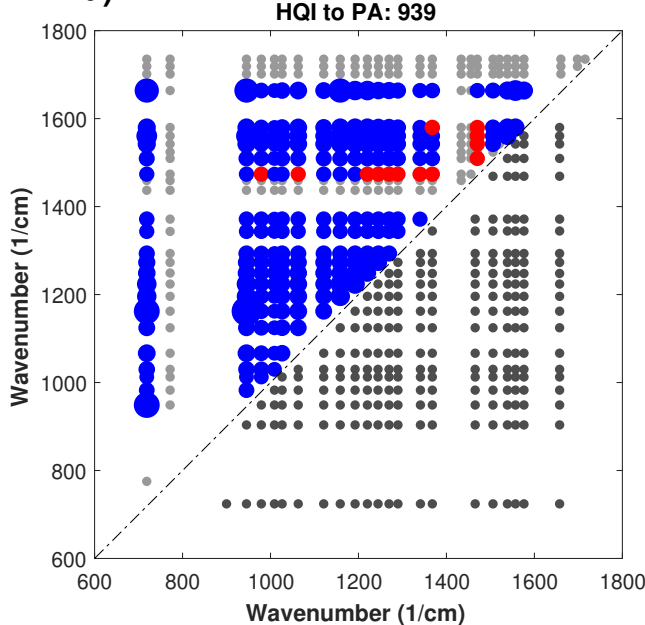

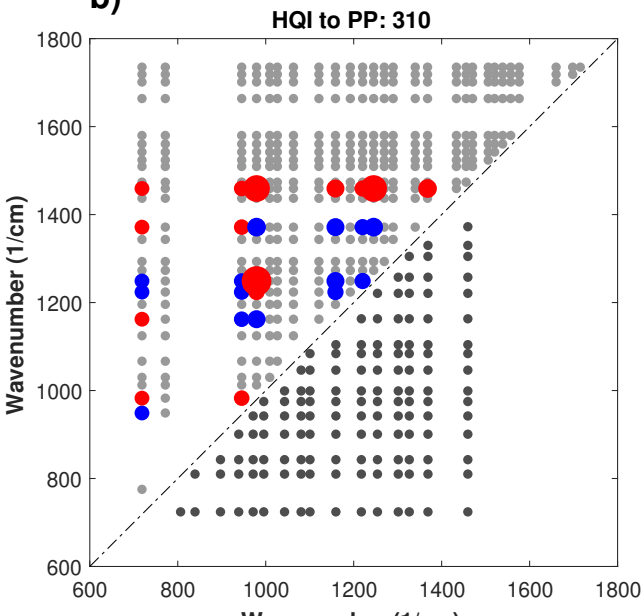

Figure S 9: Comparison of Vibrational Band Area Patterns. a) and b) show similar to Fig. 8 scatter plots of the vibrational band area patterns of a polyamide sample. However, in contrast, there are not only the triangle patterns of the sample, which can be seen above the diagonals, but there are also triangle patterns of two references below the diagonals. a) shows the comparison with a polyamide and b) with a polypropylene reference pattern. Additionally, some of the circles within the area ratios have a similarity of at lest $70 \%$ a con (the circles

\section{References}

1. Renner, G., Schmidt, T. C. \& Schram, J. A New Chemometric Approach for Automatic Identification of Microplastics from Environmental Compartments Based on FT-IR Spectroscopy. Anal. Chem. 89, 12045-12053 (2017). 


\section{HQI-Microplastics Amount Relation of Case Study 2}

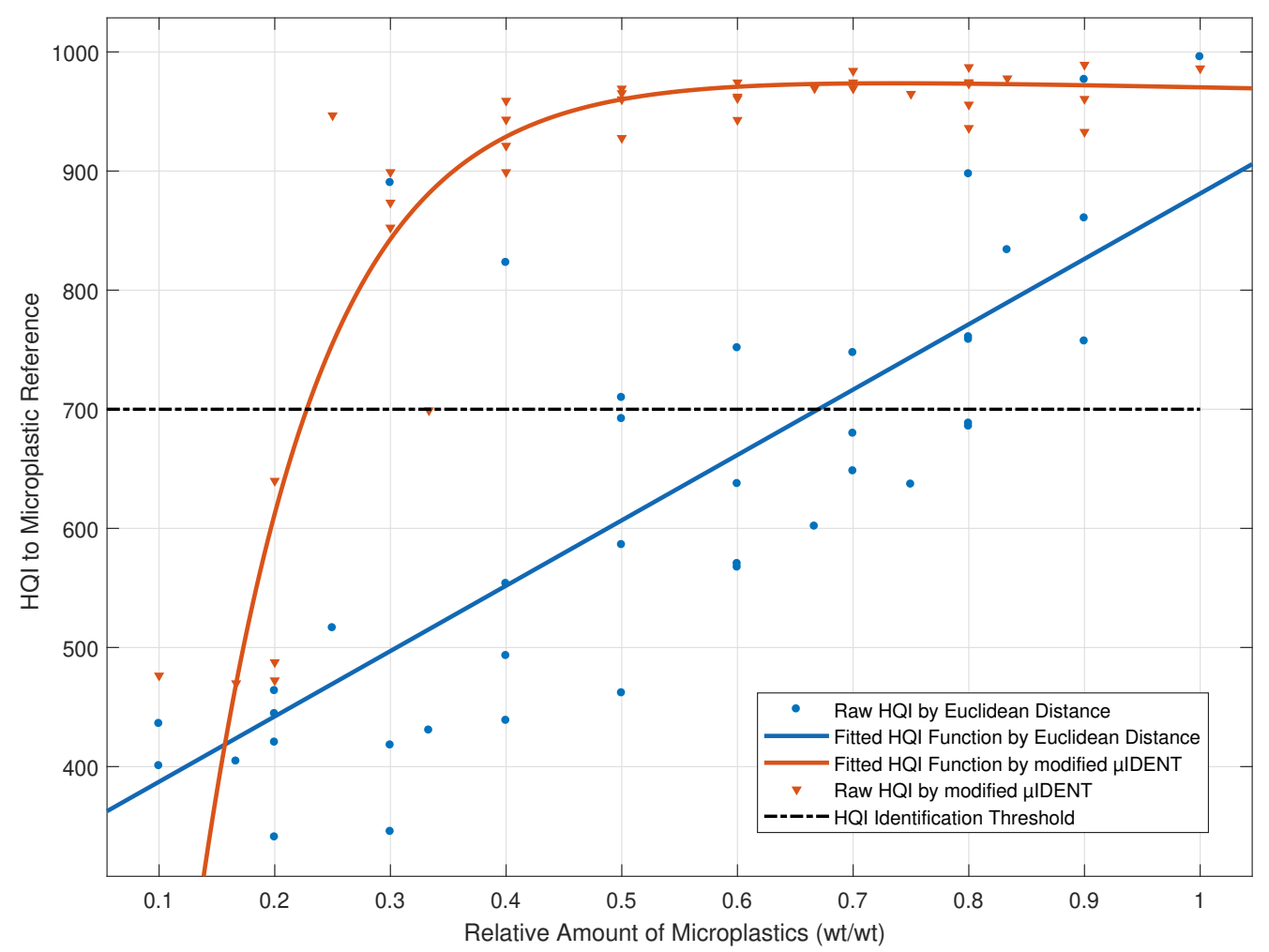

Figure S 10: Mathematical relation between the amount of microplastics and the HQI to the correct microplastic reference based on all analyzed microplastics-matrix mixtures of Case 2. The Euclidean Distance, as a representative of conventional library searching shows a linear relation between the two parameters, and a limit of automated identification can be observed at $66 \%$. In contrast, the modified $\mu$ IDENT approach shows a much more robust exponential relation and the limit of automated identification can be observed at $22 \%$. 


\section{Identification Results of Case Study 3}

All analyzed samples including the real samples from Case Study 3 were used to develop and validate the new microplastics identification algorithm (modified $\mu I D E N T$ ). The individual identification results of Case Study 3 can be obtained from Tab. 4. They agree with other studies. ${ }^{1-5}$

Table S 4: Reference Identification Results of Case Study 3. All n=1117 spectra were evaluated manually by experts.

\begin{tabular}{|lccccccccc} 
& PA & PE & PLA & PMMA & POM & PP & PS & PU & PVC \\
Case 3a $(\mathbf{n = 3 0 0})$ & 21 & 87 & 3 & 1 & 1 & 131 & 36 & 17 & 3 \\
Case 3b $(\mathbf{n = 8 1 7})$ & 13 & 184 & 22 & 4 & 15 & 371 & 106 & 73 & 29
\end{tabular}

\section{References}

1. Zhu, J. et al. Microplastic pollution in the Maowei Sea, a typical mariculture bay of China. Sci. Total Environ. 658, 62-68 (Mar. 2019).

2. Primpke, S., Lorenz, C., Rascher-Friesenhausen, R. \& Gerdts, G. An automated approach for microplastics analysis using focal plane array (FPA) FTIR microscopy and image analysis. Anal. Methods 9, 1499-1511 (2017).

3. Hidalgo-Ruz, V., Gutow, L., Thompson, R. C. \& Thiel, M. Microplastics in the marine environment: a review of the methods used for identification and quantification. Environ. Sci. Technol. 46, 3060-3075 (2012).

4. Tiwari, M., Rathod, T., Ajmal, P., Bhangare, R. \& Sahu, S. Distribution and characterization of microplastics in beach sand from three different Indian coastal environments. Mar. Pollut. Bull. 140, 262-273 (Mar. 2019).

5. Edo, C. et al. Occurrence and identification of microplastics along a beach in the Biosphere Reserve of Lanzarote. Mar. Pollut. Bull. 143, 220-227 (June 2019). 


\section{Source Code of modified $\mu I D E N T$}

Create Main Window

Listing 1: python source code

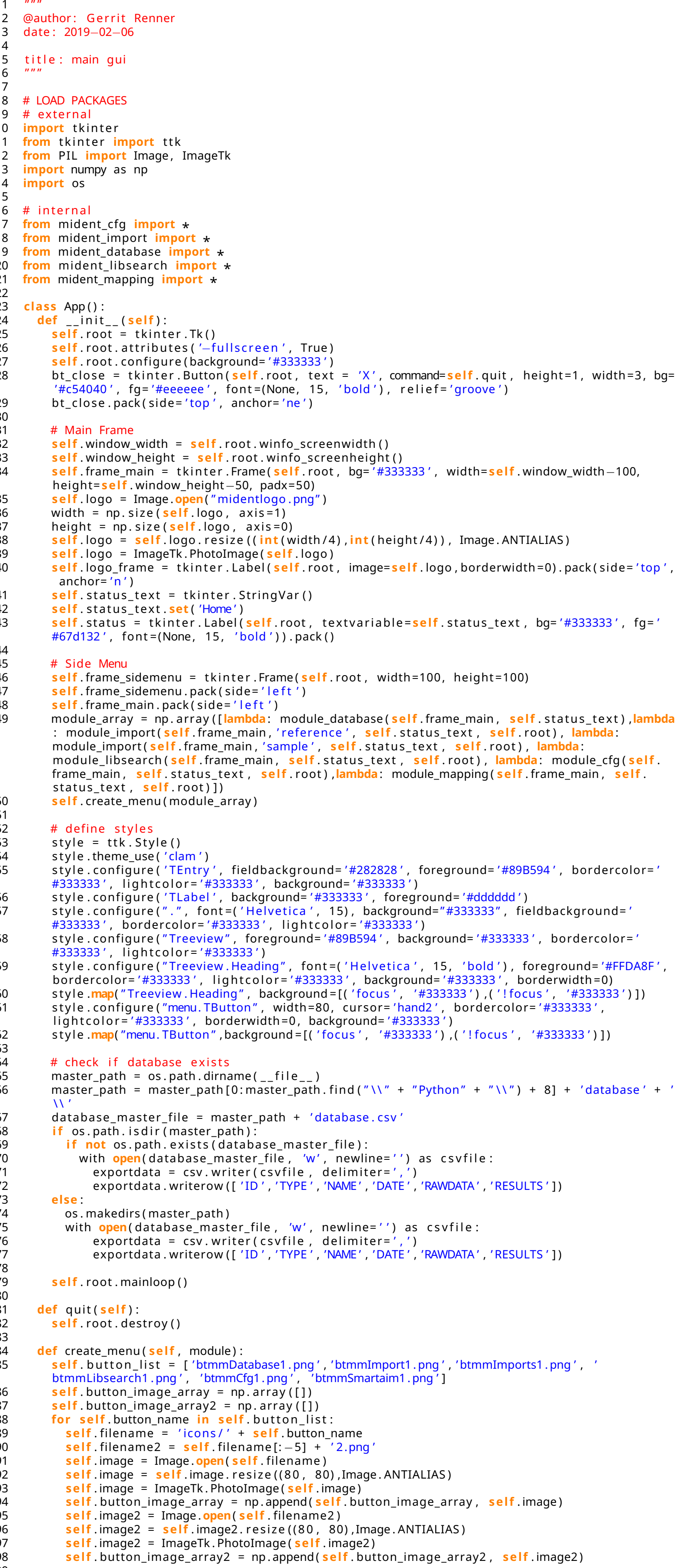

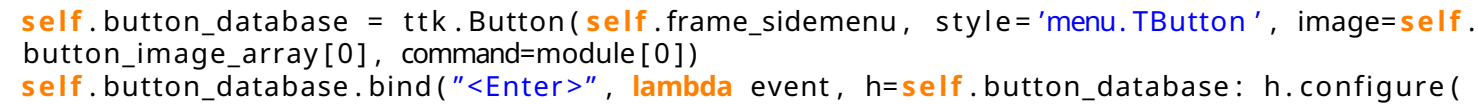

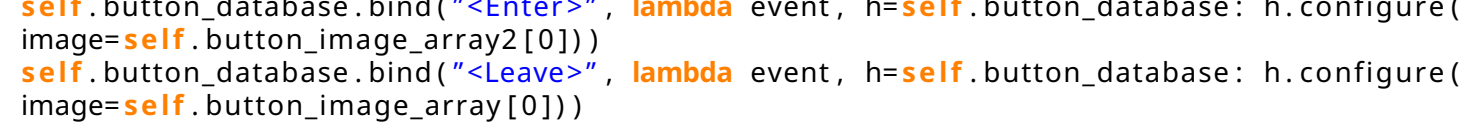

self. button_importref $=\mathrm{ttk}$. Button $(\mathrm{self}$. frame_sidemenu, style $=$ 'menu. TButton', image=self

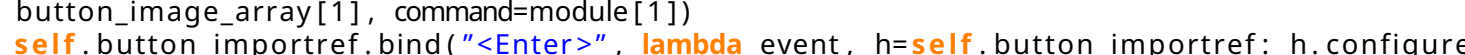

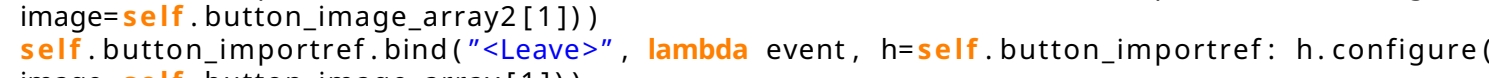

self. button_importsmp $=$ ttk. Button (self. frame_sidemenu, style='menu. TButton', image=self. button_image_array [2], command=module 2$]$ )
self. button_importsmp. bind ( $<$ Enter $>$ ", lambda event, h=self. button_importsmp: h. configure

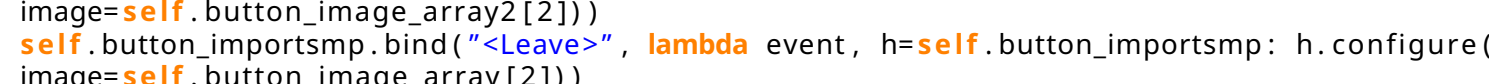

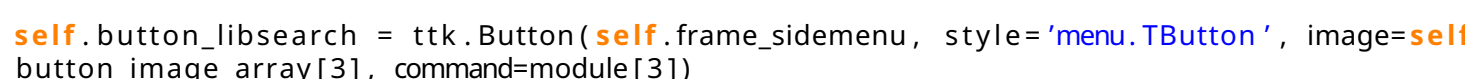
self. button_libsearch. 'bind ("<Enter>" ", lambda event, h=self. button_libsearch: h. configure

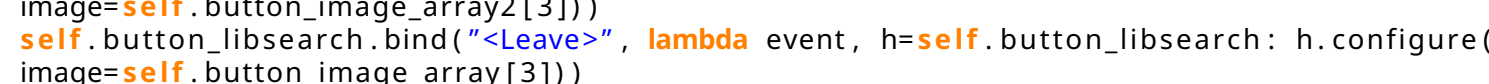
image=self. button_image_array [3])

self. button_cfg $=$ ttk. Button (self. frame_sidemenu, style='menu. TButton', image=self.

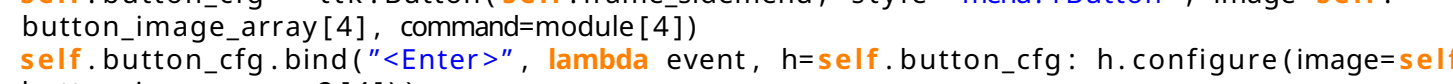

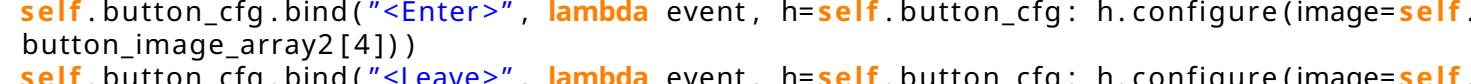
self.button_cff. bind ("<Leave>", lambda event, $h=$ self. button_cfg: h. configure (image=self.
button_image_array [4])

self. button_mapping = ttk. Button (self. frame_sidemenu, style='menu. TButton', image=self.

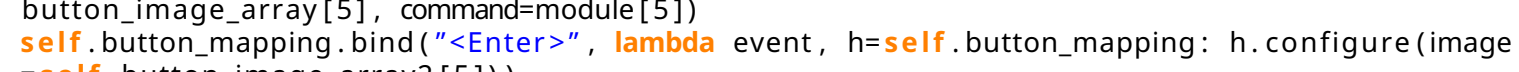
=self. button_image_array2 $2[5]))$
self. button_mapping.bind ("<Leave>", lambda event, h=self. button_mapping: h. configure (image

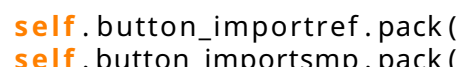

self. button database. pack ()

self. button_cfoearch.pack ()

self. button_mapping pack() 


\section{Source Code of modified $\mu I D E N T$ Import Module}

Listing 2: python source code

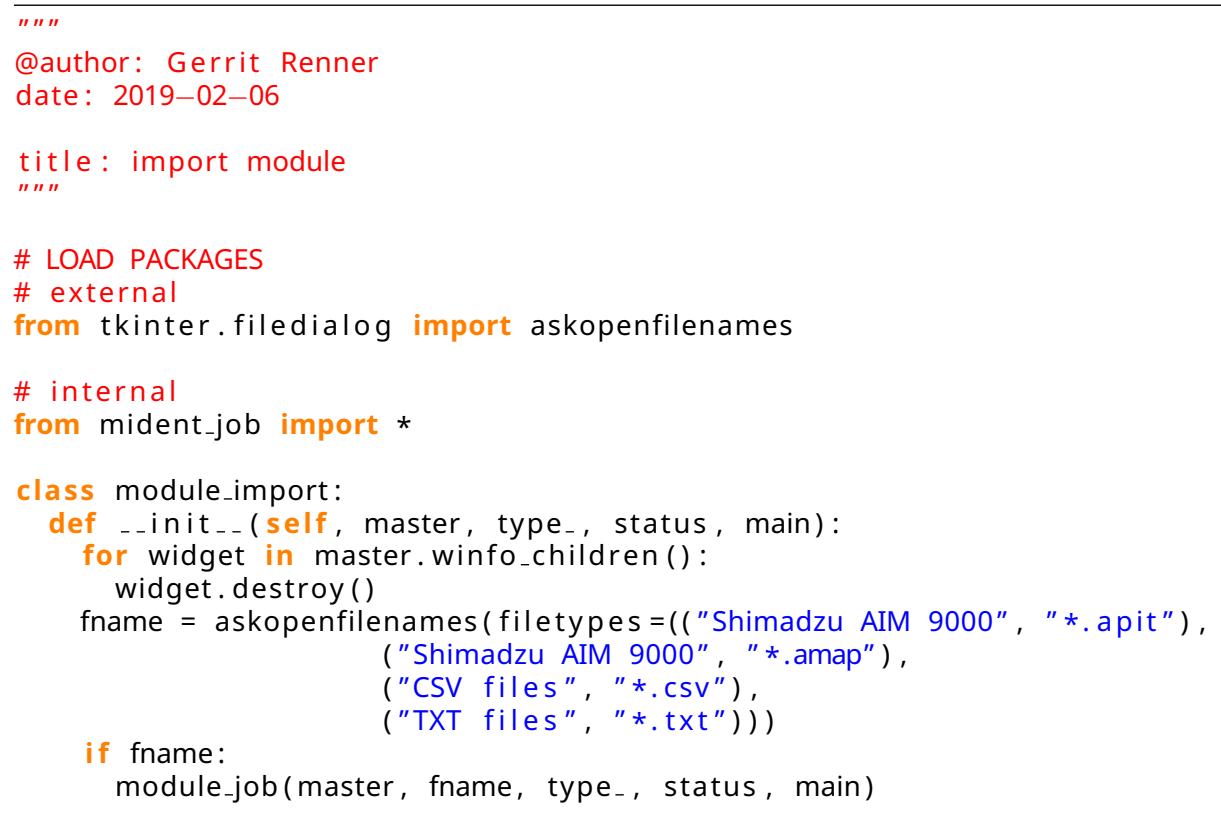




\section{Source Code of modified $\mu I D E N T$}

Listing 3: python source code

@author: Gerrit Renner
date: $2019-02-06$

title: job module

\# LOAD PACKAGES
\# external

from datalutil. parser import parse
from tkinter import ttk

from thinter import ttk
import tkinter

14 \#internal
15
16
11

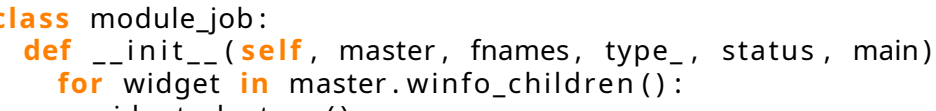

for widget in
widget. des
\# get entries

tems $=[]$
for item in fnames:
tmp type $=$ type

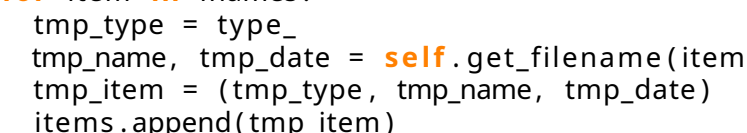

\# create table
table-header $=[$ 'TYPE', 'FILE NAME', 'DATE']
colum width $=$. 150 ,

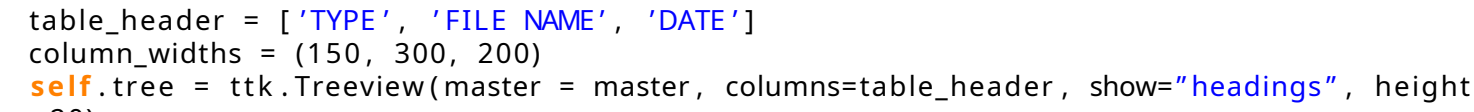

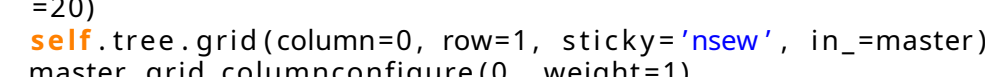

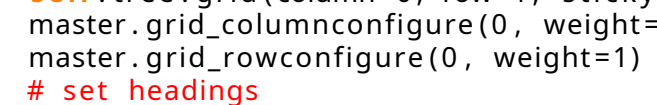

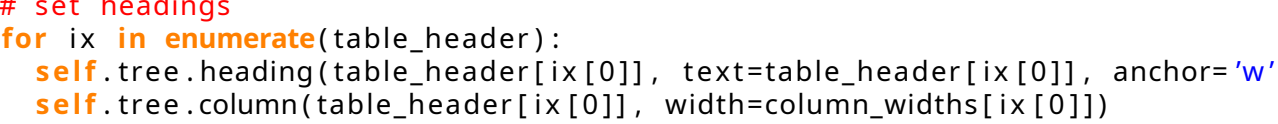

\# set items
for item in titems: (", 'end', valuesitem
self. tree. insert(",

self. tree. bind ("CDouble-1>", self. OnDoubleclick)
"create

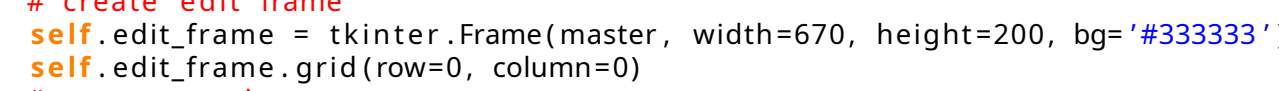

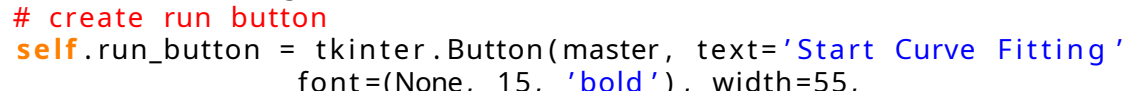

elief =' 'groove', bg=' $\$ 317256^{\prime}$

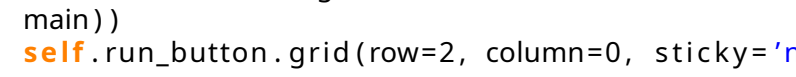

def get_filename(self, filename)

flag = 0
while flag $=$

ix = filename. find(')'

$\begin{aligned} & \text { if ix } !=-1: \\ & \text { filename }\end{aligned}=$ filename $[i x+1:]$

$\begin{aligned} \text { flag } & =1 \\ \text { \# delete ending } & \end{aligned}$

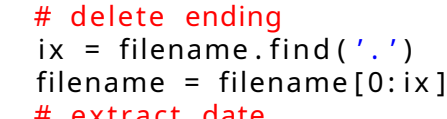

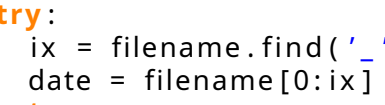

tmp $=$ int (date [0])
month $=, "$ (

month

tmp
except:

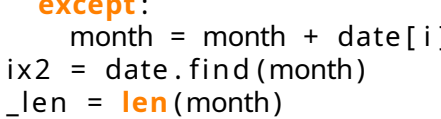

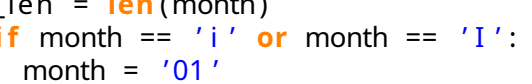

lif month $==$, 'ii' or month $==$ 'II',

month $=y^{\prime} 02$,
lif month $==$

elif month

or month $==$ ' $\mathrm{V}$

lif month
month $=$

month $=, 10, x^{\prime}$ or month $==x^{\prime}$

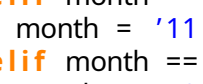

year $=$ date $[0:[1 \times 2]$
day $=\operatorname{date}\left[1 \times 2+\_\right.$len:]

if len (date) $)=6$ :
year $=120^{\circ}+$ date $[0: 22$

month $=$ date $22: 4]$
day $=$ date $[4: 6]$

year $=' 2015 '$
month $=108^{\prime}$
day $=101$ '

date $=s \operatorname{str}($ year $)+{ }^{+}-{ }^{\prime}+$ month $+{ }^{\prime}-'+\operatorname{str}($ day
name $=$ filename $[i x+1:]$

flag = False

date $=$ '2015-8-1:
name $=$ filename

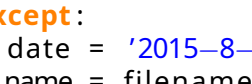

date $=$ ' $2015-8-1$,
name $=$ filename
flag $=$ True

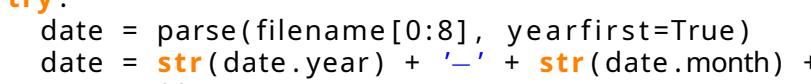

h) $+{ }^{-}-{ }^{\prime}+\operatorname{str}($ date. day

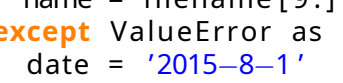

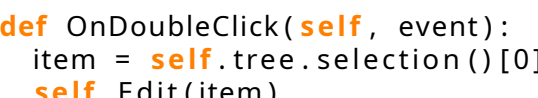

def Edit( (s elf, item):
for widget in self.
widget dit_frame. winfo_children ():

\# read item

Item_dict $=$ self. $\operatorname{tree}$. item (iter $)$
item_ID $=$ item

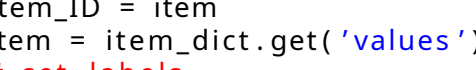

self.type _bl = ttk. Label (self. edit frame, text=' 'type

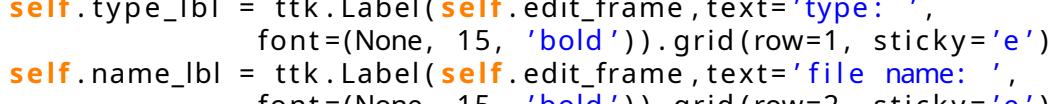

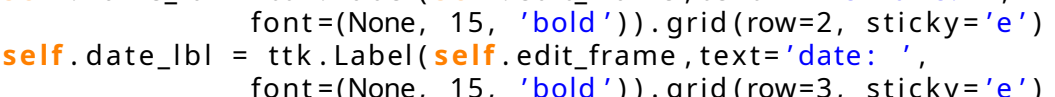

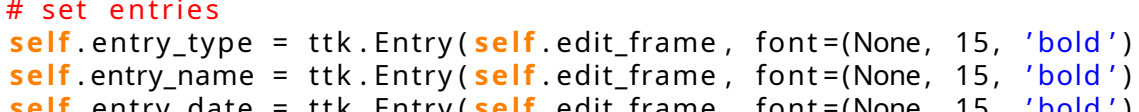

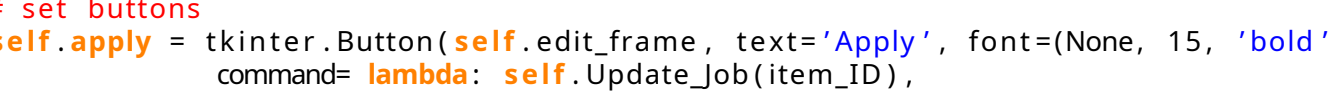

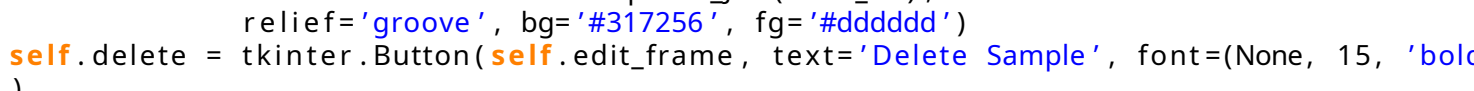

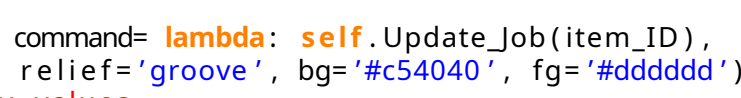

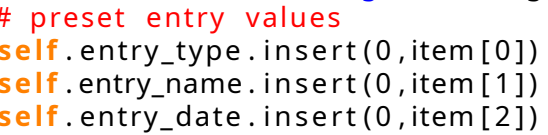

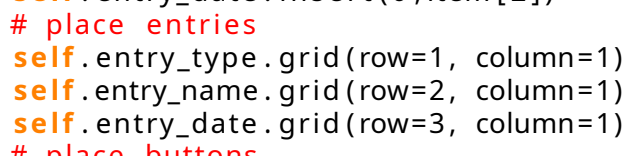

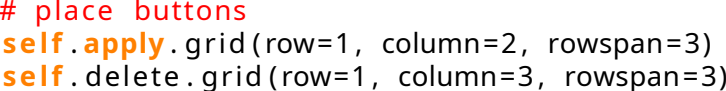

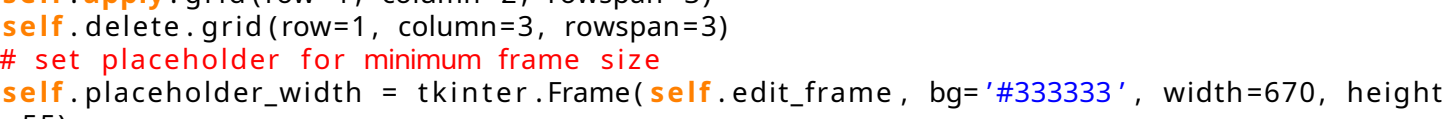

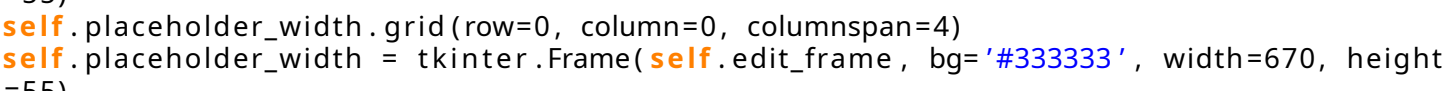

self. placeholder_width. grid (row $=4$, column $=0$, columnspan $=4$

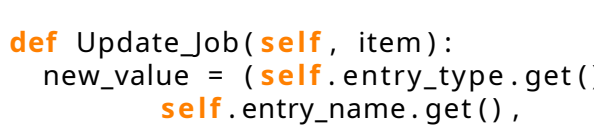

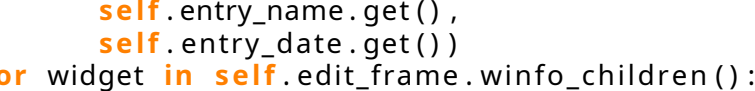

widget destroy() (),
self. tree iftem (item, values=new_value) 
E분

5

$=$

$=$

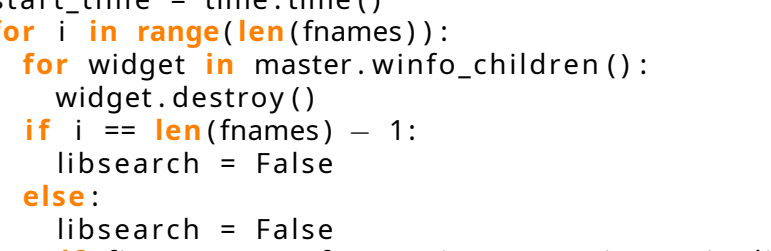

$=5$

$=$

$=$

$=$

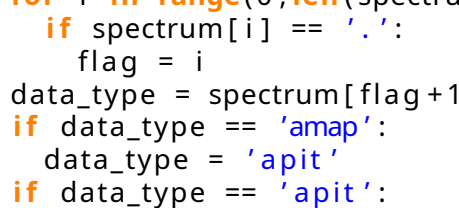

$y=$

3

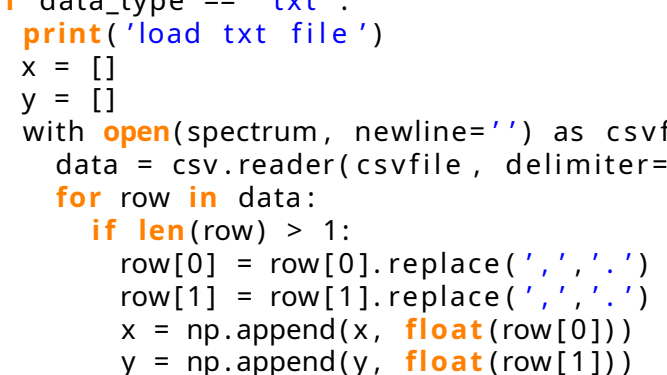

in:

$-2$

$3=$
$z=$

$=$

$=$

$=$

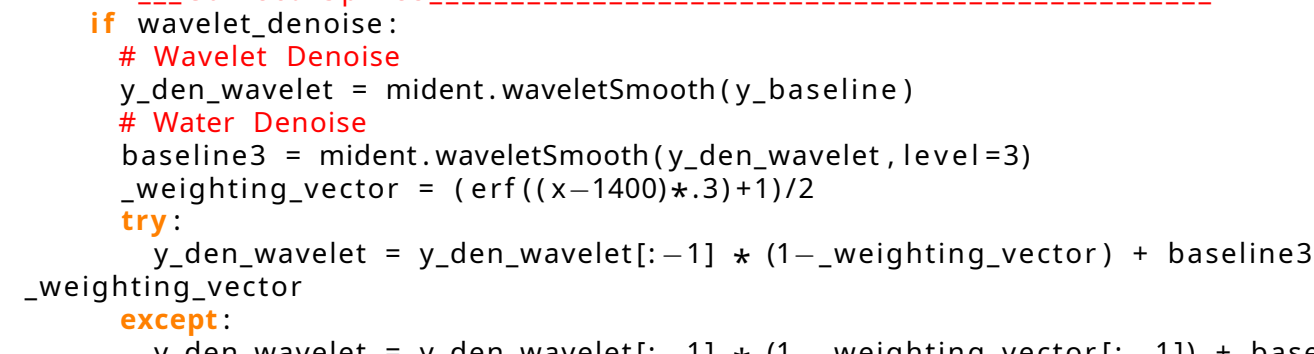

s.

$=-\cdots$

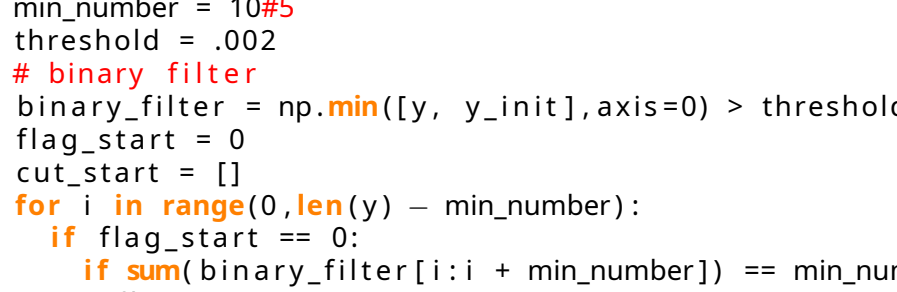

$=$

$\sum_{=10}$

ㄴ.?

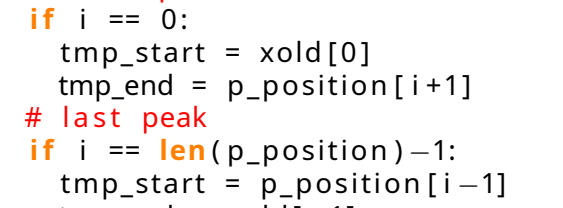

$=12=$

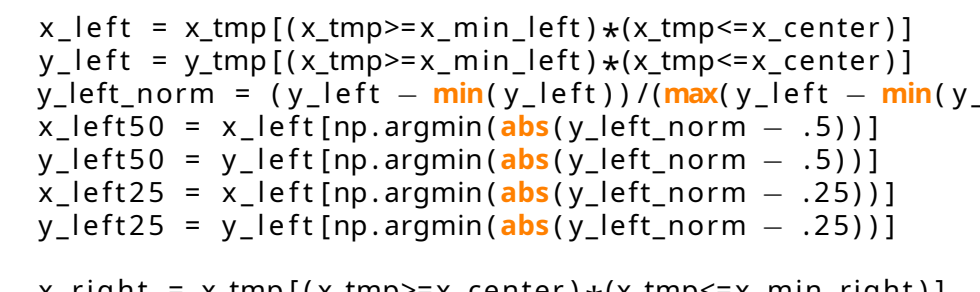

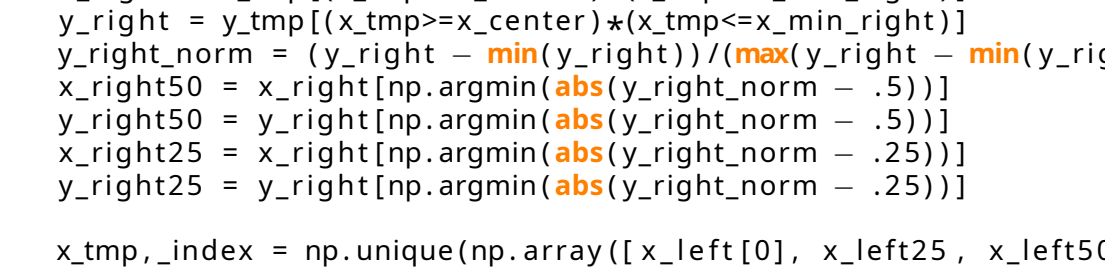

and

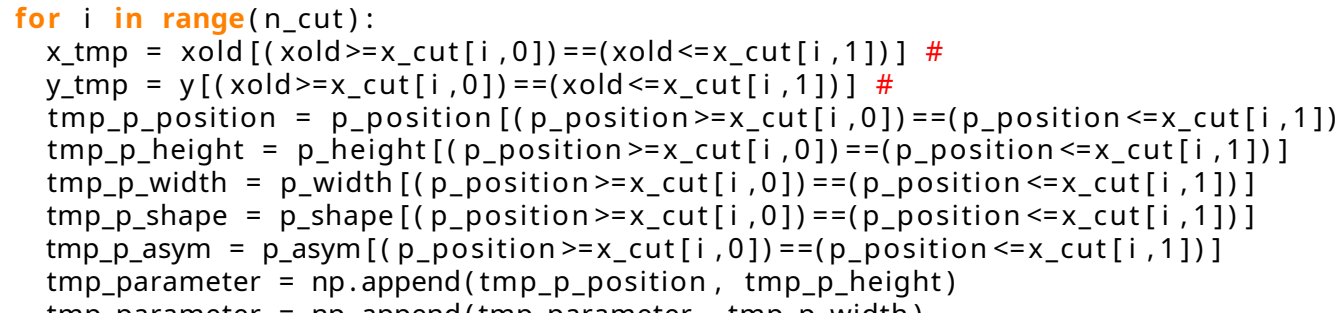

$=:=$

and

$a^{3}$

(1)

$1=$

3

$=3$

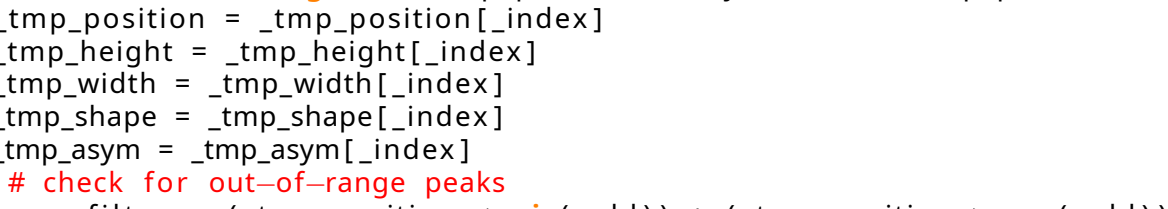

$\equiv=$

a

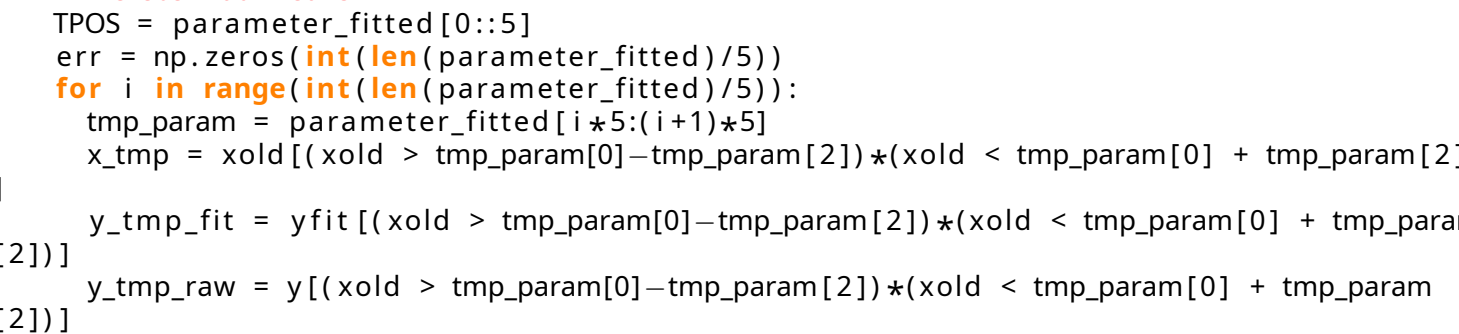

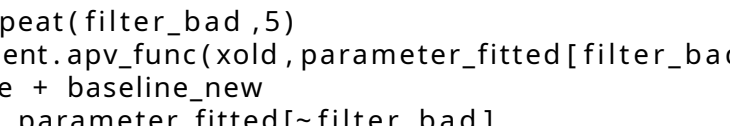

-

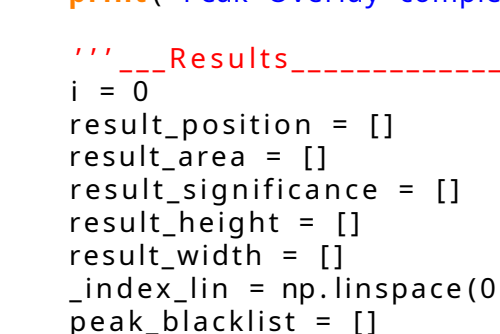

$=$

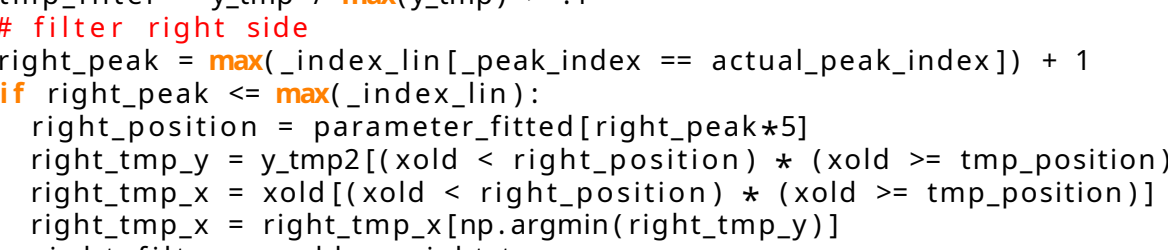

sint

$=3$

$=3$

$=$

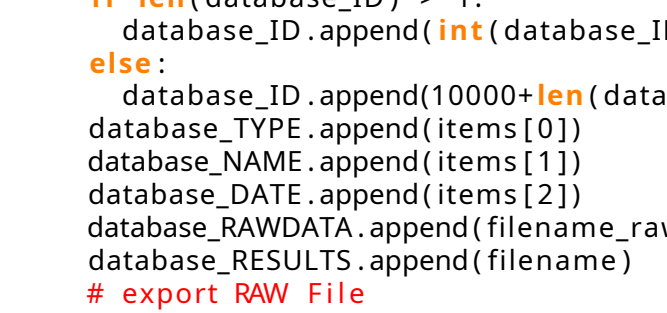

$\equiv=$

$\equiv=$

$=$

$=$

=

$=$

$= \pm=$

$=3$

$=3$

$=-m$ 


\section{Source Code of modified $\mu$ IDENT}

Database Module

Listing 5: python source code

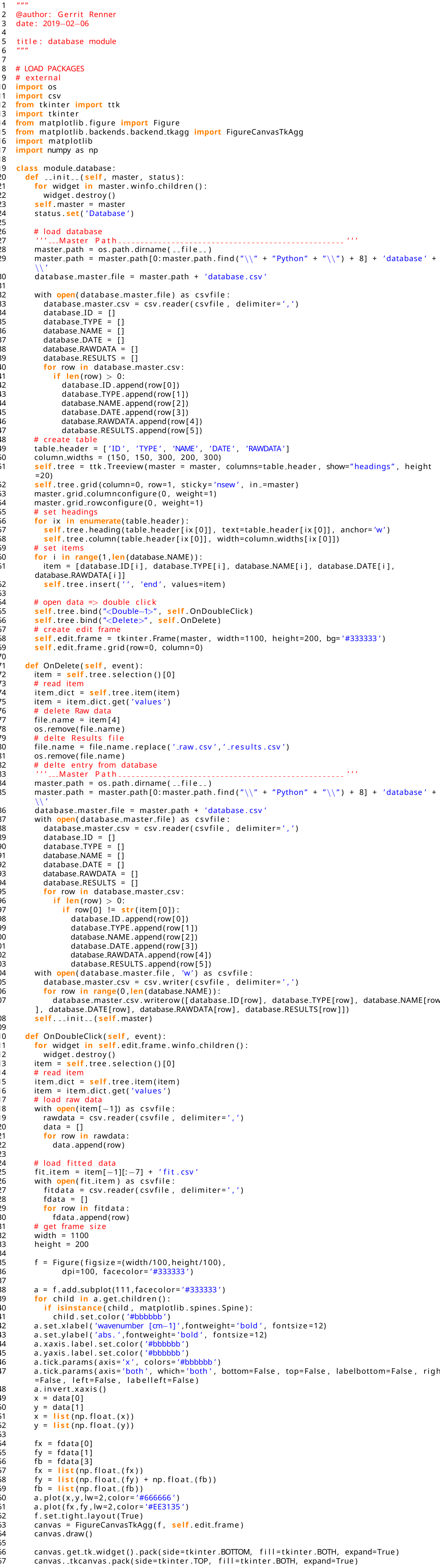




\section{N"n"
@author: Gerrit Renn
date: 2019-02-06}

title: library search module

\# LOAD PACKAGES
9 \# External

\# external
import csv
import tkinter

12 import nimper as np

np. seterr (divide $e^{\prime}$ 'ignore', invalide' 'ignore ')
from tkinter. filedialog import askopenfilenaants

class module_libsearch:
def - init_- (s self, master, status, main)
for widget in master. winfo_children ():

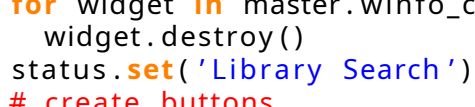

self. load_references-button_text $=$ tkinter. Stringvar ()
self. load_references_button-text set('Load References

self. load_references_button $=$ tkinter. Button (master, textvariable $=$ self.

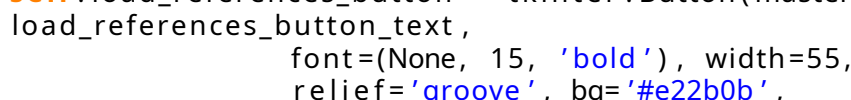

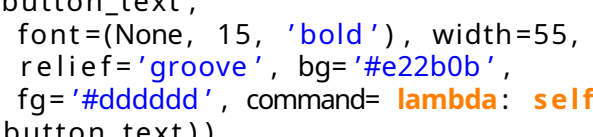

self. load_samples_button_text $=$ tkinter. Stringvar()
self. load_samples_button_text. set('Load Samples')

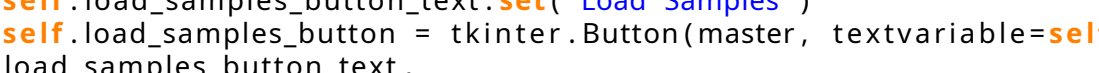

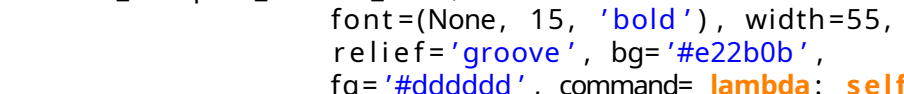

load_samples_button_text))

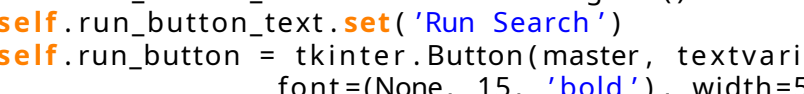

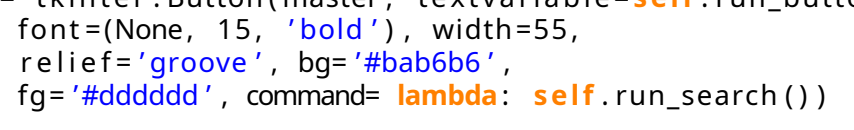

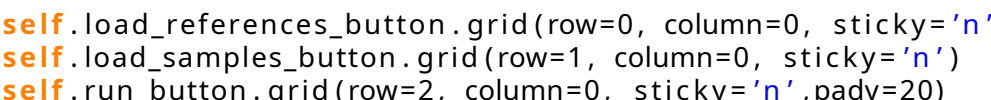

def load_references(self, button):
self.,ref_files $=$ askopenfilenames (filetypes $=\left(\left({ }^{\prime}\right.\right.$ "Reference files

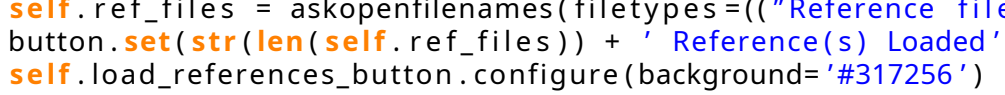

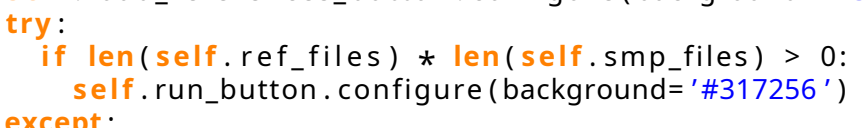

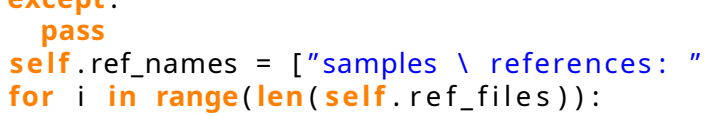

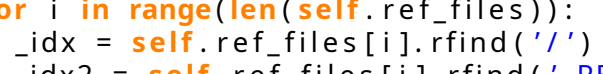

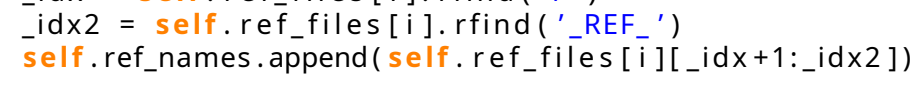

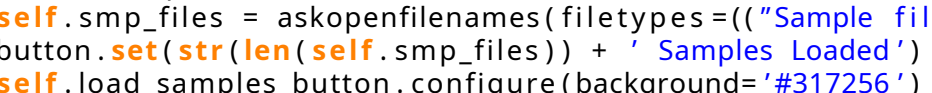

try:
if len (self. ref_files) $*$ len (self. smp_files) $>0$ :

excep:

pass
self smp_names $=[$ [
for i in range (len

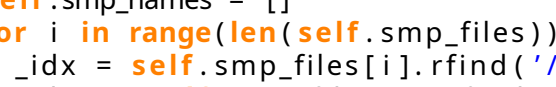

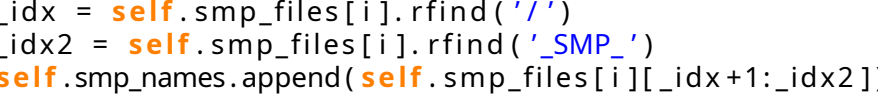

def run_search(self):

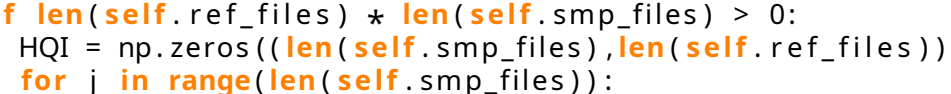

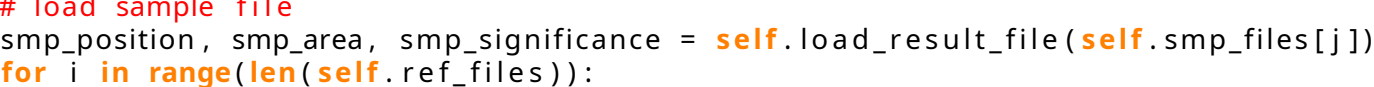

\# load reference file
ref_position, ref_area, ref_significan

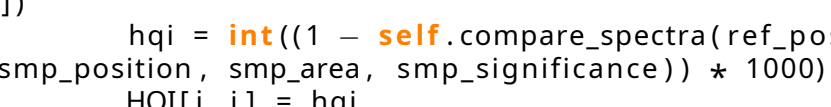

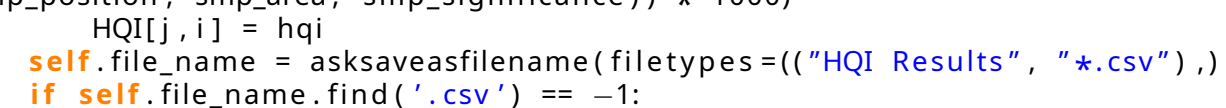

self. file_name = sel.

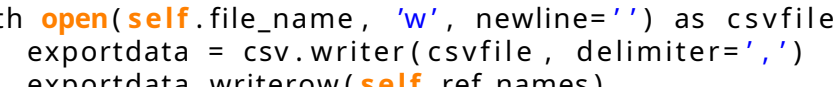

\#exportdata. Writerow (self smp.names)

row $=[$ self.smp_names[i $[i]]$,

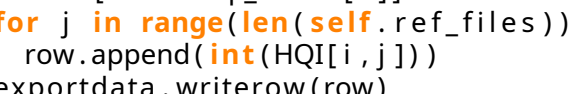

except:
pass

def load_result_file(self, fname)

with open(fframe) as csvfile:
result_file $=$ csv. reader(csvfile, delimiter=',

for row in result_file:

data. append(row
position $=$ data $[0]$

area $=$ data $[1] 1$
significance $=$

significance $=$ data $[2]$
position $=$ Iist (np. float_(position)

area $=$ list (np. float_(area))
significance = list (np. float_(significancen)

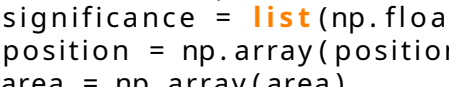

area =np. array (area)
significance $=$ np. array (significance)
return position, area, significance

def compare_spectra(self, ref_position, ref_area, ref_significance, smp_position, smp_area,

omp sind similar peaks
threshold $=10$
the

threshold $=$
flag = True
posi $=$ np. a

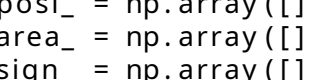

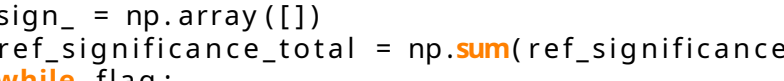

dist_ $x=n$.

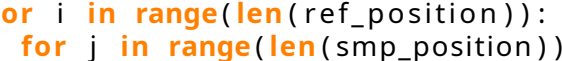

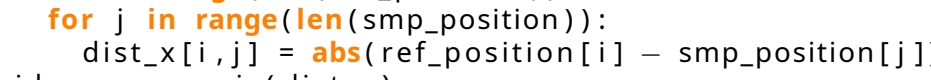

idx $=$ np. arminin (dist. $x$ )
min_min $=$ np.min (dist $-x$

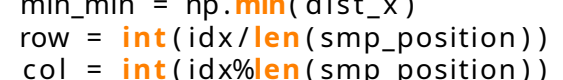

posi- = np.append(posi_, [ ref_position [row], smp_position [ col ]])

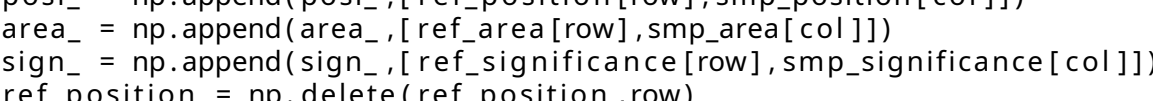

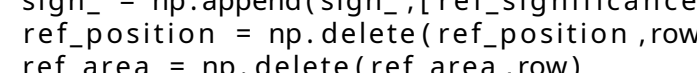

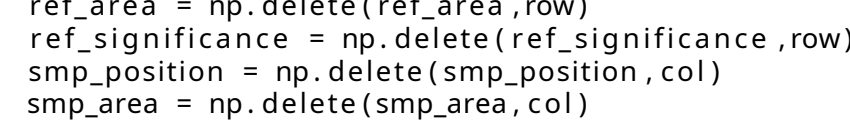

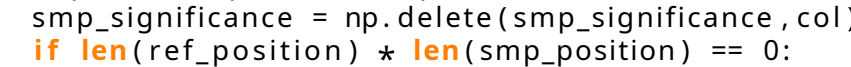

flag $=$ False
if $\min$ min $>$ threshold:

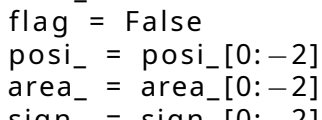

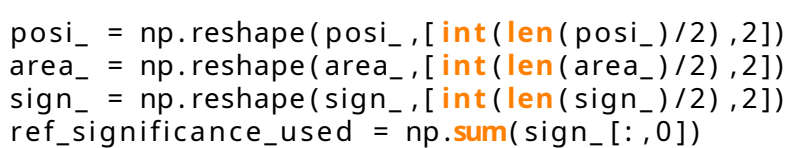

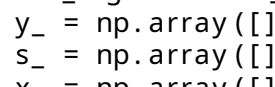

$y_{-}=$in rapgen $(i+1,1$, ap $($ posi $i)$ ):

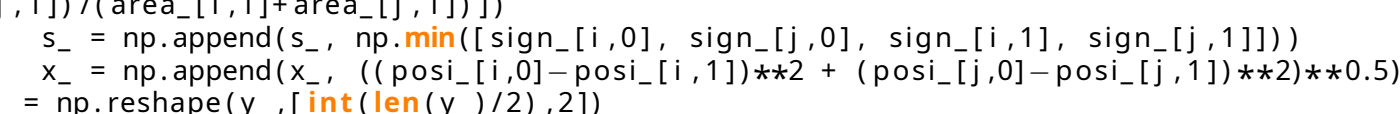

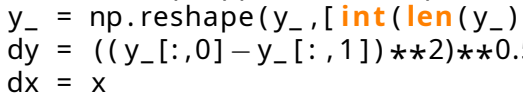

$\mathrm{k} 1=1.2$ \# Empirical factors
$\mathrm{k} 2=15$ \# Empirical factors

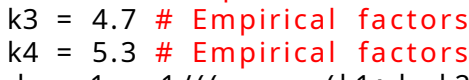

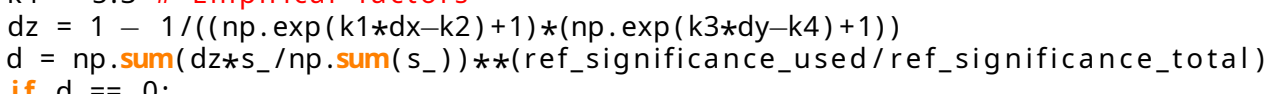

if $d==0$
$d=1$ 


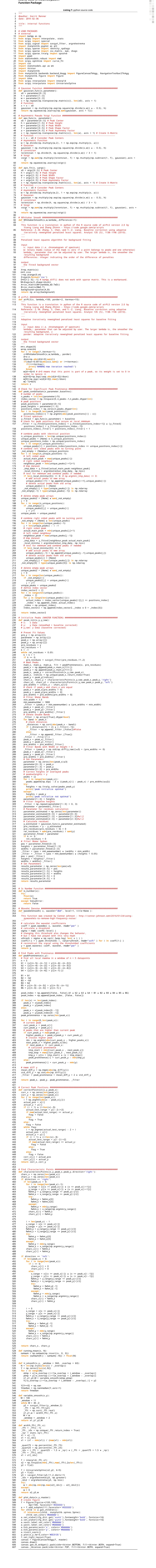


Source Code of modified $\mu I D E N T$

Graphics
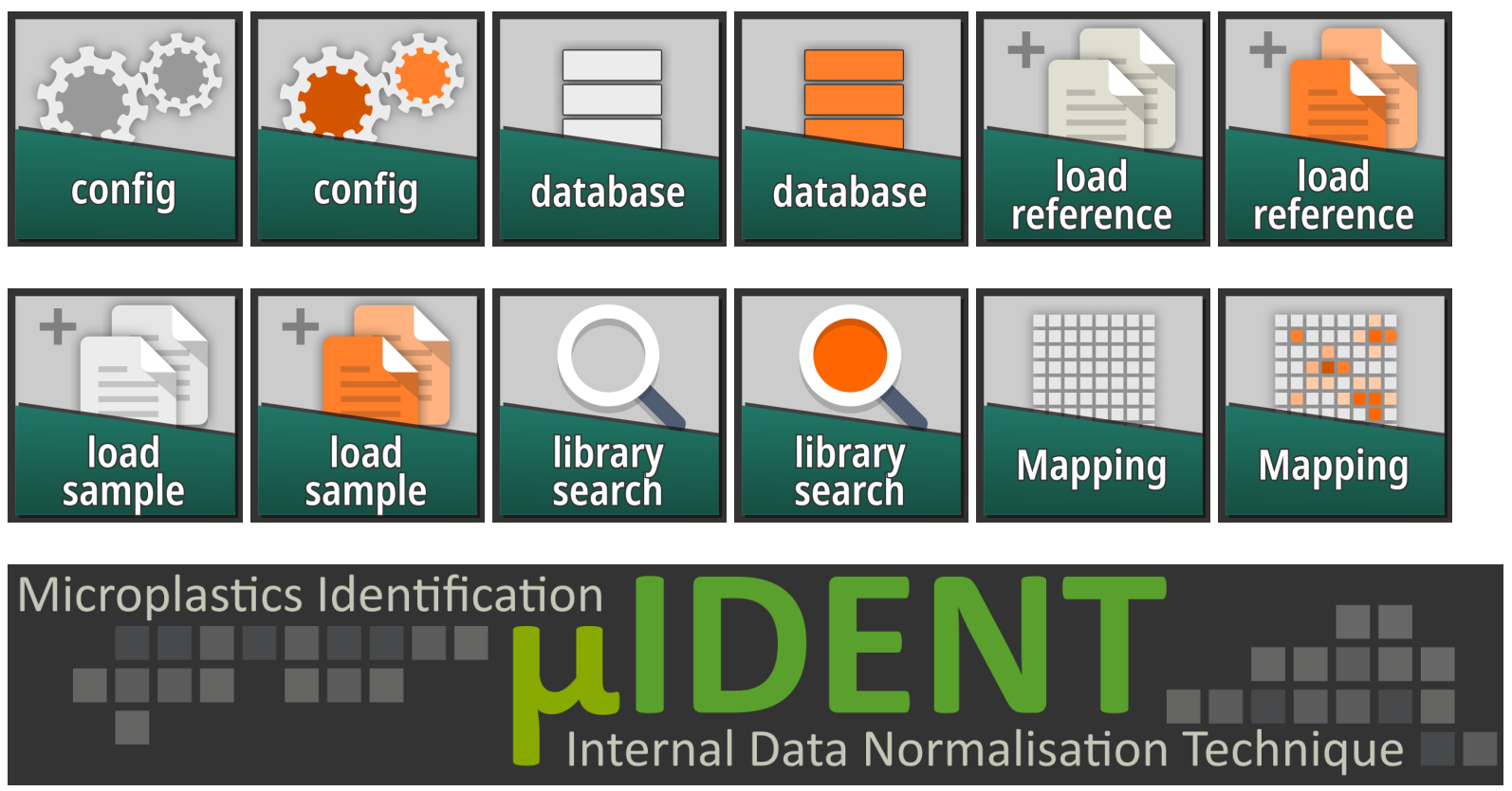